\title{
Extreme-Value Statistics of Fractional Brownian Motion Bridges
}

\author{
Mathieu Delorme and Kay Jörg Wiese \\ CNRS-Laboratoire de Physique Théorique de l'Ecole Normale Supérieure, \\ PSL Research University, Sorbonne Universités, UPMC, 24 rue Lhomond, 75005 Paris, France.
}

\begin{abstract}
Fractional Brownian motion is a self-affine, non-Markovian and translationally invariant generalization of Brownian motion, depending on the Hurst exponent $H$. Here we investigate fractional Brownian motion where both the starting and the end point are zero, commonly referred to as bridge processes. Observables are the time $t_{+}$the process is positive, the maximum $m$ it achieves, and the time $t_{\max }$ when this maximum is taken. Using a perturbative expansion around Brownian motion $\left(H=\frac{1}{2}\right)$, we give the first-order result for the probability distribution of these three variables, and the joint distribution of $m$ and $t_{\max }$. Our analytical results are tested, and found in excellent agreement, with extensive numerical simulations, both for $H>\frac{1}{2}$ and $H<\frac{1}{2}$. This precision is achieved by sampling processes with a free endpoint, and then converting each realization to a bridge process, in generalization to what is usually done for Brownian motion.
\end{abstract}

\section{INTRODUCTION}

Stochastic processes are a powerful tool to describe the evolution of systems where the microscopic dynamics is not accessible. As an example, Brownian motion, aka the Wiener process, was introduced as an effective probabilistic description for the dynamics of a particle subjected to collisions with its environment [1], be it a gas or a liquid.

An important class of such processes, which contain Brownian motion, are Markov chains. For these the evolution depends only on the current position, but is independent of previous ones. Stated differently: In a discrete dynamics the increments between successive positions are independent random variables. This Markov property is at the center of powerful tools [2] for stochastic processes, as Green-function methods, the Fokker-Plank equation, etc.

Though Markov chains successfully model many systems, there are also relevant systems which do not evolve with independent increments, and thus are non-Markovian, i.e. history dependent. Such processes naturally appear for the dynamics of a single point in a spatially extended object, as for instance a single spin in a magnet, or a marked monomer in a polymer. Their dynamics becomes non-Markovian due to the coupling to the neighbors.

Dropping the Markov property, but keeping the other ingredients of Brownian motion, i.e. Gaussianity, scale invariance and stationarity of the increments defines an enlarged class of random processes, known as fractional Brownian motion (fBm), and parameterised by the Hurst parameter $H$, which quantifies the self-affinity of the process. Its covariance function is

$$
G^{-1}\left(t_{1}, t_{2}\right)=\left\langle X_{t_{1}} X_{t_{2}}\right\rangle=t_{1}^{2 H}+t_{2}^{2 H}-\left|t_{1}-t_{2}\right|^{2 H} .
$$

Since the process is Gaussian, Eq. (1) defines it. Such processes appear in a broad range of contexts: Anomalous diffusion [3], diffusion of a marked monomer inside a polymer [4] 5], polymer translocation through a pore [5-8], singlefile diffusion [9. 11] observable experimentally in ion channels [12, 13], the dynamics of a tagged monomer [14, 15], finance (fractional Black-Scholes, fractional stochastic volatility models, and their limitations) [16-18], hydrology [19, 20], and many more. Their extreme-value statistics has been stud-

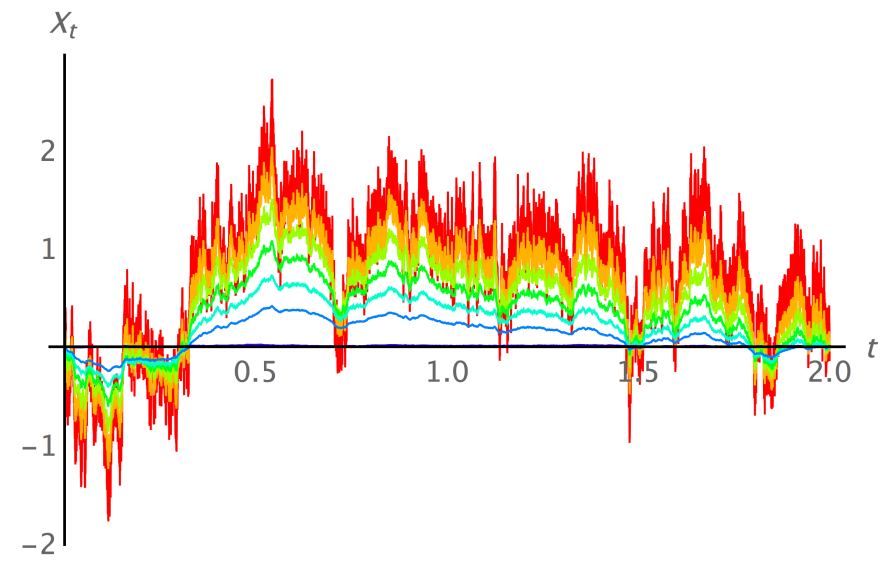

FIG. 1. Exemples of fBm bridges for different values of $H$, generated from the same random numbers using the Davis and Harte procedure [27]. $H=0.25$ in red (outmost curves) to $H=0.875$ in blue (innermost), with increments of $1 / 8$.

ied in many referenes [9,-11, 21,-26].

When studying random processes in a time interval $[0, T]$, quite generally the initial value $X_{0}$ is known, and the endpoint $X_{T}$ is itself a random variable determined by the random process. On the other hand, there are also cases when one knows the endpoint $X_{T}$. These processes are referred to as bridge processes or bridges. For a Brownian one refers to Brownian bridges.

Using a Fourier decomposition with the same amplitude for each mode, but different values of $H$, one can generate realizations of $\mathrm{fBm}$ bridges, and study their dependence on $H$, see Ref. [27] and section II below. Sample trajectories ranging from $H=0.25$ (red) to $H=0.875$ (blue) in increments of 0.125 are presented on Fig. 1

Bridges are useful building blocks in constructing more complicated observables; we will see an application of this idea in section IV below. They are also commonly used in constructing refinements of random walks, e.g. for financial modeling [28]. Finally, they appear as the difference from the asymptotic limit in the construction of the empirical distribution function [29].

We investigate three observables relevant for bridges: 
(i) the time $t_{\max }$ the random process achieves its maximum,

(ii) the value $m$ of this maximum,

(iii) the time $t_{+}$the process is positive, aka its positive time, supposing one starts at $X_{0}=0$.

For Brownian motion, and for a bridge terminating at its starting point after time $T$, both $t_{\max }$ and $t_{+}$have a uniform distribution [30]

$$
\mathcal{P}_{H=1 / 2}^{\text {bridge }}\left(t_{\text {max }}\right)=\mathcal{P}_{H=1 / 2}^{\text {bridge }}\left(t_{+}\right)=\frac{1}{T} .
$$

In contrast, for Brownian motion with a free endpoint (i.e. without constraint) the corresponding probability reads [2, 30]

$$
\mathcal{P}_{H=1 / 2}^{\text {free }}\left(t=t_{\text {max }}\right)=\mathcal{P}_{H=1 / 2}^{\text {free }}\left(t=t_{+}\right)=\frac{1}{\pi \sqrt{t(T-t)}} .
$$

These two results, as well as a way to interpolate between them for the positive-time distribution can be found in Ref. [31]. For the maximum value $m$, up to time $T$, the probability distributions are

$$
\begin{aligned}
& \mathcal{P}_{H=1 / 2}^{\text {bridge }}(m)=\frac{2 m}{T} e^{-\frac{m^{2}}{T}} \Theta(m), \\
& \mathcal{P}_{H=1 / 2}^{\text {free }}(m)=\frac{e^{-\frac{m^{2}}{4 T}}}{\sqrt{\pi T}} \Theta(m) .
\end{aligned}
$$

Properties of fractional Brownnian motion were recently investigated within a perturbative approach in $H=1 / 2+\varepsilon$, expanding around Brownian motion, corresponding to $H=1 / 2$ [32, 34]. We extend these results by considering bridge processes. While observables related to the maximum of an unconstrained fractional Brownian motion were already considered in Refs. [33, 34], the observable $t_{+}$is considered for the first time here. Indeed, we will show that at leading order in $\varepsilon=H-\frac{1}{2}$, the probability distributions for $t_{\max }$ and $t_{+}$are different, contrary to Brwonian motion, and processes with a free endpoint, where they agree at leading order [35].

Finally we test our analytical results against numerical simulations for $H=0.4, H=0.6$, and $H=0.66$. This is achieved by constructing a subtracted process out of each realization of a fBm with free endpoints. This procedure yields the same statistics as a fractional Brownian bridge, and is much more efficiently simulated than an unconstrained $\mathrm{fBm}$, for which one retains only realizations which are bridges.

This article is organised as follows: Section II introduces some general results about Gaussian bridges, as well as their application to fractional Brownian motion.

Section III recalls the methodology developed in Ref. [34] on the perturbative expansion around Brownian motion.

Section IV introduces $t_{+}$, the time spent by the process in the positive half space. We start with a discrete random walk before taking the continuum limit to obtain the distribution of $t_{+}$for Brownian motion. This is used as a starting point for the perturbative expansion described in the previous section, with some technical steps left to appendix B. The analytical results obtained are then compared to numerical simulations.

Section $\mathrm{V}$ presents results on the extreme-value statistics for a fBm bridge: the maximum value $m$ as well as the time $t_{\max }$ to reach it. Some of these results are derived from a general calculation performed in Ref. [34]; we also present a new and simpler way to obtain the maximum-value distribution.

Several appendices complete our work: Appendix B contains details about the inverse of an integral transform appearing in our calculation, and its relation to the Abel transform.

Appendix C summarises the necessary inverse Laplace transforms needed in the main text.

\section{PRELIMINARIES: GAUSSIAN BRIDGES}

Consider a real-valued process $X_{t}$, starting at $X_{0}=0$. We define a bridge, denoted $X_{t}^{\mathrm{B}}$, to be the same process conditioned to be at $a$ at time $T$. Its one- and two-point correlation functions are

$$
\begin{aligned}
\left\langle X_{t_{1}}^{\mathrm{B}}\right\rangle & =\frac{\left\langle X_{t_{1}} \delta\left(X_{T}-a\right)\right\rangle}{\left\langle\delta\left(X_{T}-a\right)\right\rangle}, \\
\left\langle X_{t_{1}}^{\mathrm{B}} X_{t_{2}}^{\mathrm{B}}\right\rangle & =\frac{\left\langle X_{t_{1}} X_{t_{2}} \delta\left(X_{T}-a\right)\right\rangle}{\left\langle\delta\left(X_{T}-a\right)\right\rangle} .
\end{aligned}
$$

We now assume that $X_{t}$ is a centered Gaussian process, i.e. $\left\langle X_{t}\right\rangle=0$ for all $t$, and that cumulants of order higher than 2 vanish. To express the correlation function of the bridge process in terms of the unconditioned process, we insert the identity $\delta(x)=\int_{-\infty}^{\infty} e^{i k x} \frac{\mathrm{d} k}{2 \pi}$ into the above equations. After some lines of algebra presented in appendix A we arrive at

$$
\begin{aligned}
\left\langle X_{t_{1}}^{\mathrm{B}}\right\rangle & =a \frac{\left\langle X_{t_{1}} X_{T}\right\rangle}{\left\langle X_{T}^{2}\right\rangle} \\
\left\langle X_{t_{1}}^{\mathrm{B}} X_{t_{2}}^{\mathrm{B}}\right\rangle & =\left\langle X_{t_{1}} X_{t_{2}}\right\rangle-\left[\left\langle X_{T}^{2}\right\rangle-a^{2}\right] \frac{\left\langle X_{t_{1}} X_{T}\right\rangle\left\langle X_{t_{2}} X_{T}\right\rangle}{\left\langle X_{T}^{2}\right\rangle^{2}} .
\end{aligned}
$$

Consider now the subtracted process $X_{t}^{\mathrm{S}}$ defined from the original process $X_{t}$ as

$$
X_{t}^{\mathrm{S}}:=X_{t}-\left(X_{T}-a\right) \frac{\left\langle X_{t} X_{T}\right\rangle}{\left\langle X_{T}^{2}\right\rangle}
$$

One easily checks that its one and two-point correlation functions coincide with those of $X_{t}^{\mathrm{B}}$ given in Eqs. (8)- 9]. This is sufficient to conclude that $X_{t}^{\mathrm{B}}$ and $X_{t}^{\mathrm{S}}$ are the same processes,

$$
X_{t}^{\mathrm{S}} \stackrel{\text { law }}{=} X_{t}^{\mathrm{B}}
$$

While this result was derived in Ref. [36] by other methods, the prescription (10) does not seem to be generally known.

Frequently used for Brownian motion $X_{t}:=B_{t}$ the subtracted process 10$]$ reduces to

$$
B_{t}^{\mathrm{S}}=B_{t}-\frac{t}{T}\left(B_{T}-a\right) .
$$

This is equivalent in law to a Brownian bridge ending at $a$. 
For fractional Bronwian motion with Hurst exponent $H$, the subtracted term is non-linear in $t$, containing the expression

$$
f\left(\vartheta:=\frac{t}{T}\right):=\frac{\left\langle X_{t} X_{T}\right\rangle}{\left\langle X_{T}^{2}\right\rangle}=\frac{1}{2}\left[1+\vartheta^{2 H}-(1-\vartheta)^{2 H}\right] .
$$

The equivalence 111 is crucial for the numerical simulations presented in this work. Simulating bridge process using its definition requires to discard almost all generated paths, while the subtracted process can be constructed from every generated path without loss of statistics.

\section{PERTUBATIVE APPROACH TO FBM}

We recall here some useful definitions for $\mathrm{fBm}$, as well as the ideas of the perturbative expansion around Brownian motion developed in Refs. [32, 34].

First, as fractional Brownian motion is a Gaussian process, it is characterized by its covariance function $G^{-1}$ given in Eq. (1). This covariance function allows us to write an action for the possible realizations of $X_{t}$,

$$
S[X]=\frac{1}{2} \int_{t_{1}, t_{2}} X_{t_{1}} G\left(t_{1}, t_{2}\right) X_{t_{2}} .
$$

This yields the average of any observable $O[X]$ for the $\mathrm{fBm}$ within a path-integral formulation,

$$
\langle O[X]\rangle=\int \mathcal{D}[X] O[X] e^{-S[X]} .
$$

To compute observables explicitly from this expression, we expand the action around Brownian motion, corresponding to $H=1 / 2$ in Eq. (1). Writing $H=1 / 2+\varepsilon$, we obtain at first order in $\varepsilon$

$$
\begin{aligned}
S[X]= & \int_{0}^{T} \mathrm{~d} t_{1} \frac{\dot{X}_{t_{1}}^{2}}{4 D_{\varepsilon, \tau}} \\
& -\frac{\varepsilon}{2} \int_{0}^{T-\tau} \mathrm{d} t_{1} \int_{t_{1}+\tau}^{T} \mathrm{~d} t_{2} \frac{\dot{X}_{t_{1}} \dot{X}_{t_{2}}}{\left|t_{2}-t_{1}\right|}+\mathcal{O}\left(\varepsilon^{2}\right) .
\end{aligned}
$$

The first term is the standard action of Brownian motion, with a rescaled diffusive constant

$$
D_{\varepsilon, \tau}=1+2 \varepsilon[1+\ln (\tau)]+O\left(\varepsilon^{2}\right) .
$$

The regularisation cut-off $\tau$ (wich is an UV cut-off in time) appears in the second term of the action, which is a non-local (in time) interaction between derivatives of the process. For the derivation of this expansion we refer to Ref. [32].

Note that the non-locality in time of the action is a manifestation of the non-Markovian nature of fractional Brownian motion. We will use this formalism to compute observables for bridges of $\mathrm{fBm}$ in an $\varepsilon$ expansion, following the strategy and using results of Ref. [34].

\section{POSITIVE TIME OF A FBM BRIDGE}

In this section, we investigate the distribution of the time spend up to time $T$ by the process $X_{t}$ in the positive half space. This time, denoted $t_{+}$, is defined by

$$
t_{+}:=\int_{0}^{T} \mathrm{~d} t \Theta\left(X_{t}\right)
$$

where $\Theta$ is the Heavyside function, $\Theta(x)=1$ if $x>0$, and $\Theta(x)=0$ otherwise, and $X_{0}=0$.

Below, we first consider a discrete random walk and derive the Laplace transform (i.e. generating function) of the distribution of $t_{+}$. Taking the continuous-time limit allows us to obtain the distribution of $t_{+}$for Brownian motion. We use this result to construct our perturbative expansion for a fractional Brownian motion bridge, and to derive an analytical prediction at order $\varepsilon$.

\section{A. Positive time of a discrete random walk}

Consider a discrete random walk $X_{n}$ with discrete steps \pm 1 (without bias), starting at $X_{0}=0$. We denote $N_{n, x}$ the number of paths which goes from 0 to $x$ in $n$ steps. This number is non-zero only if $x$ and $n$ have the same parity and $x$ is smaller than $n$. It can be obtained by retaining the term of order $q^{x}$ from the generating function for all paths, $\left(q+q^{-1}\right)^{n}$, i.e.

$$
\left(q+\frac{1}{q}\right)^{n}=\sum_{i=0}^{n} q^{i}\left(\frac{1}{q}\right)^{n-i}\left(\begin{array}{c}
n \\
i
\end{array}\right) .
$$

Identifying $x=2 i-n$ yields

$$
N_{n, x}=\left(\begin{array}{c}
n \\
\frac{n+x}{2}
\end{array}\right) \text {. }
$$

It can also be deduced as follows: Paths ending in $x$ have $n_{+}=\frac{n+x}{2}$ up segments, and $n_{-}=\frac{n-x}{2}$ down segments. The number of paths with $n_{+}$up segments is $\left(\begin{array}{c}n \\ n_{+}\end{array}\right)$, which again yields Eq. 20p.

Denote by $N_{n, x}^{+}$the number of strictly positive paths, i.e. $X_{i}>0$ for all $i>0$, which go from 0 to $x>0$ in $n$ steps. By the reflexion principle, illustrated on figure 2 this is the same as the number of paths that go from 1 to $x$ in $n-1$ steps, minus the number of paths which start at -1 and go to $x$ in $n-1$ steps,

$$
N_{n, x}^{+}=N_{n-1, x-1}-N_{n-1, x+1}=\frac{x}{n} N_{n, x} .
$$

The ratio

$$
\frac{N_{n, x}^{+}}{N_{n, x}}=\frac{x}{n}
$$




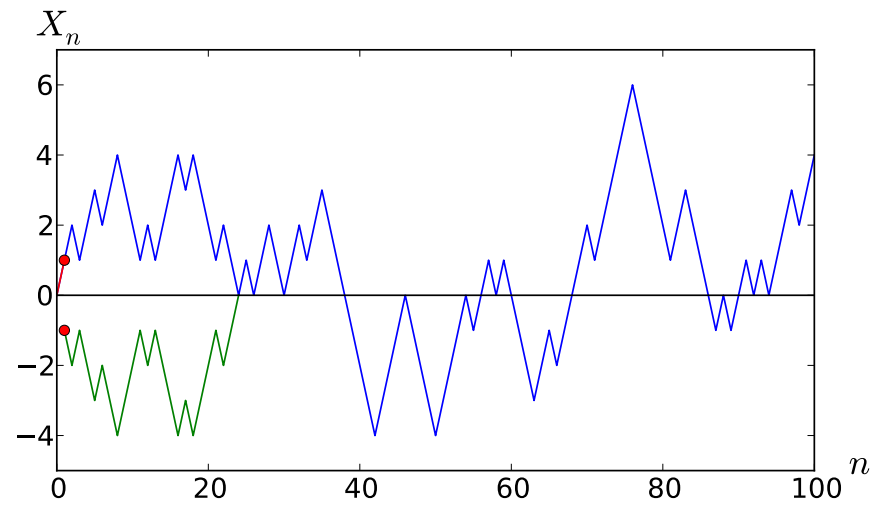

FIG. 2. Illustration of the reflection principle: Every path emanating from 1 and attaining zero again (blue) is compensated by a reflected path emanating from -1 (green).

is the probability that a path from 0 to $x$ in $n$ steps is strictly positive, also known as the Ballot theorem ${ }^{1}$.

Another quantity of interest is the number of excursions, i.e. paths that go from $X_{0}=0$ to $X_{2 n}=0$ with all intermediate positions positive, and which we denote $N_{2 n}^{+ \text {,first }}$, because the end point is the first zero of the path. Such a path necessarily has $X_{2 n-1}=1$, which gives

$$
N_{2 n}^{+, \text {first }}=N_{2 n-1,1}^{+}=\frac{1}{2 n-1}\left(\begin{array}{c}
2 n-1 \\
n
\end{array}\right)=\frac{(2 n-2) !}{n !(n-1) !} .
$$

We now study the time when a random process is positive: A segment $S_{i}$ from $i-1$ to $i$ is considered positive if $X_{i-1}+$ $X_{i}>0$, and negative otherwise. Note that contrary to the positions $X_{i}$, a segment $S_{i}$ is either positive or negative. The time $t_{+}$a random walk is positive is defined as the number of positive segments.

Denote $N_{2 n, 2 k}^{\text {pos }}$ the number of bridge paths of length $2 n$ with $2 k$ positive intervals; by convention we set $N_{0,0}^{\text {pos }}:=1$. We can use Eq. 23) to get a recursion relation for $N_{2 n, 2 k}^{\text {pos }}$, with $n \geq 1$,

$N_{2 n, 2 k}^{\mathrm{pos}}=\sum_{i=1}^{n}\left[N_{2 i}^{+,{ }^{\text {first }}} N_{2(n-i), 2(k-i)}^{\mathrm{pos}}+N_{2 i}^{+, \text {first }} N_{2(n-i), 2 k}^{\mathrm{pos}}\right]$.

This is illustrated on figure 2 In this sum, $2 i$ is the position of the first zero (after the origin) of the path of lenght $2 n$. Since the path does not change sign these $2 i$ first segments are either all positive (first term inside the sum) or negative (second term).

To solve this equation, we introduce two generating func-

\footnotetext{
${ }^{1}$ The ballot theorem states that if in an election candidate $A$ receives $p$ votes and candidate $B$ receives $q$ votes with $p>q$, the probability that $A$ stays ahead of $B$ throughout the count is $(p-q) /(p+q)$, see Refs. [2] 37].
}

tions:

$$
\begin{aligned}
\tilde{p}^{\mathrm{pos}}(\nu, \rho) & :=\sum_{n \geq 0} \sum_{k \geq 0} \nu^{2 k} \rho^{2 n} \frac{N_{2 n, 2 k}^{\mathrm{pos}}}{2^{2 n}}, \\
\tilde{p}^{+, \text {first }}(\rho) & :=\sum_{n>0} \rho^{2 n} \frac{N_{2 n}^{+, \text {first }}}{2^{2 n}}=\frac{1-\sqrt{1-\rho^{2}}}{2} .
\end{aligned}
$$

Inserting these definitions into Eq. (24) transforms the recursion relation into an algebraic equation

$$
\tilde{p}^{\text {pos }}(\nu, \rho)=\left[\tilde{p}^{+, \text {first }}(\nu \rho)+\tilde{p}^{+, \text {first }}(\rho)\right] \tilde{p}^{\text {pos }}(\nu, \rho)+1 .
$$

Eq. 27) can be solved as

$$
\tilde{p}^{\text {pos }}(\nu, \rho)=\frac{1}{1-\tilde{p}^{+, \text {first }}(\nu \rho)-\tilde{p}^{+, \text {first }}(\rho)} .
$$

This is a geometric sum of the form

$$
\tilde{p}^{\text {pos }}(\nu, \rho)=\sum_{n \geq 0}\left[\tilde{p}^{+, \text {first }}(\nu \rho)+\tilde{p}^{+, \text {first }}(\rho)\right]^{n} .
$$

Its interpretation is simple: All bridges can be constructed as a sequence of first-return bridges. In a first-return bridge each factor of $\rho$ comes with a factor of $\nu$ for the positive paths, and alone for negative paths.

Using the explicit expression of Eq. 26, we obtain

$$
\tilde{p}^{\text {pos }}(\nu, \rho)=\frac{2}{\sqrt{1-(\nu \rho)^{2}}+\sqrt{1-\rho^{2}}} .
$$

Other generating functions can be obtained as well: First, for the probability to return to zero (including the term with zero steps) the latter is

$$
\tilde{p}_{0}(\rho):=\sum_{n \geq 0} \rho^{n} \frac{N_{n, x}}{2^{n}}=\frac{1}{\sqrt{1-\rho^{2}}} .
$$

For the probability to return to 0 without having become negative, this is (including the term with zero steps)

$$
\begin{aligned}
\tilde{p}_{0}^{\geq 0}(\rho) & =\frac{1}{1-\tilde{p}_{\text {first }}^{+}(\rho)} \equiv \tilde{p}^{\text {pos }}(0, \rho) \\
& =\frac{2}{1+\sqrt{1-\rho^{2}}} .
\end{aligned}
$$

The generating function for paths starting at zero and ending in $x$ without ever returning to zero can be obtained as well

$$
\begin{aligned}
\tilde{p}_{x}^{+}(\rho) & :=\sum_{n \geq 0} \rho^{n} \frac{N_{n, x}^{+}}{2^{n}} \\
& =\frac{\rho^{x}}{\left(1+\sqrt{1-\rho^{2}}\right)^{x}}=\frac{\left(1-\sqrt{1-\rho^{2}}\right)^{x}}{\rho^{x}} .
\end{aligned}
$$

This can be understood by considering the path from the end: One can first go up and down to the starting value $x$ for a number $n \geq 0$ steps, before going down by one step, leading to $\tilde{p}_{0}^{\geq 0}(\rho) \times \frac{\rho}{2}$ for the generating function to (backwards!) reach $x-1$. Repeating this $x$ times, and using Eq. (32), we arrive at Eq. (33). 


\section{B. Propagators in continuous time}

We now wish to take the continuum limit. To this aim, we note that in the limit of a time-discretisation step $\delta t \rightarrow 0$, the process

$$
X_{t} \simeq \sqrt{2 \delta t} X_{n}, \text { with } n=\text { floor }\left(\frac{t}{\delta t}\right)
$$

converges to a Brownian. The normalisation ensure that we recover the covariance function (1) with $H=\frac{1}{2}$.

Denote by $\mathcal{P}\left(t_{+}, X_{0}=x_{1}, X_{T}=x_{2}\right)$ the probability distribution of the positive time $t_{+}$within the interval $[0, T]$ for a standard Brownian motion $X_{t}$, starting at $X_{0}=x_{1}$ and ending at $X_{T}=x_{2}$. For our perturbative expansion it is useful to have this in Laplace variables, namely

$$
\begin{aligned}
& \tilde{W}^{+}\left(\lambda, s, x_{1}, x_{2}\right) \\
& \quad=\int_{0}^{\infty} \mathrm{d} T \int_{0}^{T} \mathrm{~d} t_{+} e^{-s T-\lambda t_{+}} \mathcal{P}\left(t_{+}, X_{0}=x_{1}, X_{T}=x_{2}\right) .
\end{aligned}
$$

We now use the result from the previous section, starting with the special case $x_{1}=x_{2}=0$. The probability distribution for a Brownian that its positive time, up to time $T$, is $t_{+}$and that $X_{0}=X_{T}=0$, i.e. the process is a bridge, can be obtained from the discrete case via

$$
\left.\mathcal{P}\left(t_{+}, X_{T}\right) \mathrm{d} t_{+} \mathrm{d} X_{T}\right|_{X_{T}=0} \underset{\delta t \rightarrow 0}{\simeq} \frac{1}{2^{n}} N_{n, k}^{\text {pos }} .
$$

Here $n=$ floor $(T / \delta t), k=$ floor $\left(t_{+} / \delta t\right)$, and $\delta t$ is the time discretisation step. This allows us to relate the generating function (30) to the Laplace transform of the continuoustime distribution $\tilde{W}^{+}$with $x_{1}=x_{2}=0$, which we denote $\tilde{W}^{+}(\lambda, s)$, setting $\nu \rightarrow e^{-\delta t \lambda}, \rho \rightarrow e^{-\delta t s}$ and then taking the limit of $\delta t \rightarrow 0$. The measure $\mathrm{d} t_{+} \mathrm{d} B_{T}$ gives a factor of $\sqrt{2} \delta t^{3 / 2}$, c.f. Eq. (34). This yields

$$
\begin{aligned}
\tilde{W}^{+}(\lambda, s) \sqrt{2} \delta t^{3 / 2} & \simeq \tilde{p}^{\text {pos }}\left(e^{-\delta t \lambda}, e^{-\delta t s}\right) \delta t^{2} \\
& \simeq \frac{2 \delta t^{2}}{\sqrt{1-e^{-2 \delta t(s+\lambda)}}+\sqrt{1-e^{-2 \delta t s}}} \\
& \simeq \frac{\sqrt{2} \delta t^{3 / 2}}{\sqrt{\lambda+s}+\sqrt{s}}+\mathcal{O}\left(\delta t^{2}\right)
\end{aligned}
$$

Thus

$$
\tilde{W}^{+}(\lambda, s)=\frac{1}{\sqrt{\lambda+s}+\sqrt{s}} .
$$

From this result for the bridge we obtain the expression for $\tilde{W}\left(\lambda, s, x_{1}, x_{2}\right)$ by distinguishing two cases, see Fig. 3 . The first case is when the process changes sign at least once. It can be decomposed into a constant-sign part (contributing to $t_{+}$or not, depending on the signe of $x_{1}$ ), a bridge part, and another constant sign part ending in $x_{2}$. The other case is when the process never changes sign, which corresponds to the survival probability and can be expressed using the method of images.

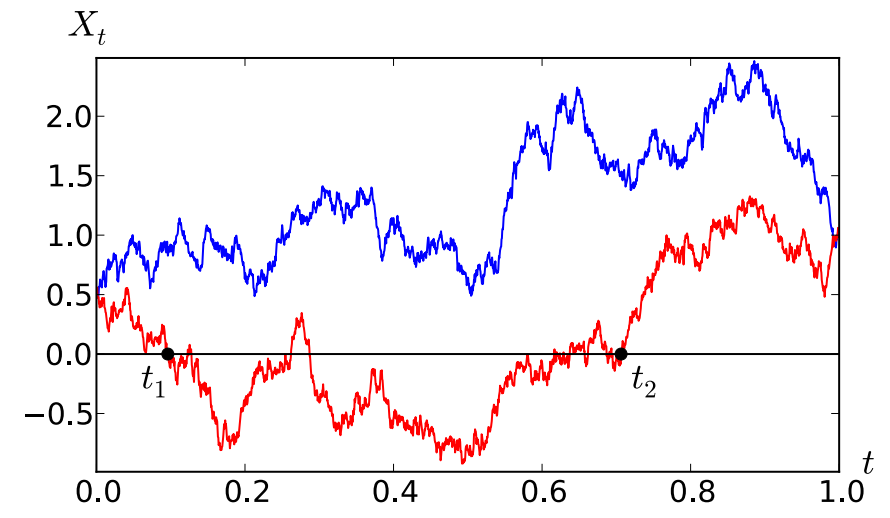

FIG. 3. In red (bottom curve) a contribution to $\tilde{W}_{1}^{+}\left(\lambda, s, x_{1}, x_{2}\right)$, where the path reaches 0 at least once (here for $x_{1}=0.5$ and $x_{2}=1$ ). In blue (top curve) the additional contribution to $\tilde{W}_{2}^{+}\left(\lambda, s, x_{1}, x_{2}\right)$, where the path never reaches 0 , possible when $x_{1}$ and $x_{2}$ have the same sign (here for $x_{1}=0.5$ and $x_{2}=1$ ).

We recall the Laplace transform of this propagator from $x_{1}$ to $x_{2}$, conditioned that the path has never touched zero [34],

$$
\tilde{P}_{0}^{+}\left(x_{1}, x_{2} ; s\right)=\frac{e^{-\sqrt{s}\left|x_{1}-x_{2}\right|}-e^{-\sqrt{s}\left|x_{1}+x_{2}\right|}}{2 \sqrt{s}} \Theta\left(x_{1} x_{2}\right) .
$$

The normalized limit $x_{1} \rightarrow 0$ is

$$
\tilde{P}_{0}^{+}\left(x_{2} ; s\right)=\lim _{x_{1} \rightarrow 0} \frac{1}{x_{1}} \tilde{P}_{0}^{+}\left(x_{1}, x_{2} ; s\right)=e^{-\sqrt{s} x_{2}} \Theta\left(x_{2}\right) .
$$

The final result is the sum of two terms,

$$
\tilde{W}^{+}\left(\lambda, s, x_{1}, x_{2}\right)=\tilde{W}_{1}^{+}\left(\lambda, s, x_{1}, x_{2}\right)+\tilde{W}_{2}^{+}\left(\lambda, s, x_{1}, x_{2}\right) .
$$

The first contribution involves a crossing, and is a product of two factors 40 and one factor 38 ,

$$
\begin{aligned}
& \tilde{W}_{1}^{+}\left(\lambda, s, x_{1}, x_{2}\right) \\
& =e^{-\sqrt{s+\lambda \Theta\left(x_{1}\right)}\left|x_{1}\right|} \frac{1}{\sqrt{s+\lambda}+\sqrt{s}} e^{-\sqrt{s+\lambda \Theta\left(x_{2}\right)}\left|x_{2}\right|},
\end{aligned}
$$

The $\Theta$ functions in the exponential are understood as follows: If $x_{1}>0$, then $s$ is changed to $s+\lambda$, since this segment contributes both to $T$ and $t_{+}$. In the opposite case $x_{1}<0$, this segment contributes only to $T$ but not to $t_{+}$, thus $s$ remains unchanged. The same argument applies to the last factor as a function of the sign of $x_{2}$.

The contribution when the walk never changes sign is

$$
\begin{aligned}
& \tilde{W}_{2}^{+}\left(\lambda, s, x_{1}, x_{2}\right) \\
& =\frac{e^{-\sqrt{s+\lambda \Theta\left(x_{1}\right)}\left|x_{1}-x_{2}\right|}-e^{-\sqrt{s+\lambda \Theta\left(x_{1}\right)}\left|x_{1}+x_{2}\right|}}{2 \sqrt{s+\lambda \Theta\left(x_{1}\right)}} \Theta\left(x_{1} x_{2}\right) .
\end{aligned}
$$

This is the propagator (39), with again $s$ shifted to $s+\lambda$ if $x_{1}$, and as a consequence also $x_{2}$, are positive. 
The result for $\tilde{W}^{+}\left(\lambda, s, x_{1}, x_{2}\right)$ can also be obtained by solving the Fokker-Planck equation

$$
\begin{aligned}
& \partial_{x_{2}}^{2} \tilde{W}^{+}\left(\lambda, s, x_{1}, x_{2}\right) \\
& \quad=\left[s+\lambda \Theta\left(x_{2}\right)\right] \tilde{W}^{+}\left(\lambda, s, x_{1}, x_{2}\right)+\delta\left(x_{1}-x_{2}\right) .
\end{aligned}
$$

One verifies that $\tilde{W}_{1}^{+}+\tilde{W}_{2}^{+}$is indeed a solution.

As a check, we consider Brownian motion starting at 0 and without any constraint at the end point, by integrating $\tilde{W}_{+}$ over the last variable

$$
\int_{-\infty}^{\infty} \mathrm{d} x \tilde{W}_{+}(\lambda, s, 0, x)=\frac{1}{\sqrt{s(s+\lambda)}} .
$$

The corresponding probability distribution for $t_{+}$is known as one of the Arcsine laws, as given in Eq. (3). Indeed, computing the double Laplace transform from this known result yields Eq. 45):

$$
\int_{0}^{\infty} \mathrm{d} T \int_{0}^{T} \mathrm{~d} t_{+} e^{-s T-\lambda t_{+}} \frac{1}{\pi \sqrt{t_{+}\left(T-t_{+}\right)}}=\frac{1}{\sqrt{s(s+\lambda)}} .
$$

For a Brownian Bridge, i.e. $x_{1}=x_{2}=0$, we have

$$
\tilde{W}^{+}(\lambda, s, 0,0)=\tilde{W}^{+}(\lambda, s)=\frac{1}{\sqrt{\lambda+s}+\sqrt{s}} .
$$

Let us note some subtleties. Eq. (47) is the double Laplace transform of the probability distribution that the Brownian process spends a time $t_{+}$in the positive half space and ends in 0 at time $T$. If we want to have the condtional probability distribution for $t_{+}$, knowing that the process is a bridge, we need to divide the result by the probability density to return to $x=0$ at time $T$, which is $(2 \sqrt{\pi T})^{-1}$. The double Laplace transform to compute is then

$$
\int_{0}^{\infty} \mathrm{d} T \int_{0}^{T} \mathrm{~d} t_{+} e^{-s T-\lambda t_{+}} \frac{1}{T} \frac{1}{2 \sqrt{\pi T}}=\frac{1}{\sqrt{\lambda+s}+\sqrt{s}} .
$$

Here $1 / T$ is the uniform probability distribution (2) of $t_{+}$for a Brownian Bridge, and $(2 \sqrt{\pi T})^{-1}$ is the probability density to return to 0 at time $T$. This indeed reproduces Eq. 477.

\section{Scale invariance and a useful transformation}

The fact that $\mathrm{fBm}$ is a scale invariant (i.e. self affine) process implies interesting properties for various distributions. For $t_{+}$, and similarly for other temporal observables, the distribution $\mathcal{P}_{H}^{T}\left(t_{+}\right)$for a fBm process defined on $[0, T]$ (with either a free end-point or a constrained one) takes the scaling form

$$
\mathcal{P}_{H}^{T}\left(t_{+}\right)=\frac{1}{T} g\left(\vartheta=\frac{t_{+}}{T}\right)
$$

Using this, the double Laplace transform of the distribution can be reformulated using a one-variable transformation:

$$
\begin{aligned}
\tilde{\mathcal{P}}_{H}(\lambda, s) & =\int_{0}^{\infty} \mathrm{d} T \int_{0}^{T} \mathrm{~d} t_{+} e^{-s T-\lambda t_{+}} \mathcal{P}_{H}^{T}\left(t_{+}\right) \\
& =\int_{0}^{\infty} \mathrm{d} T \int_{0}^{1} \mathrm{~d} \vartheta e^{-T(s+\lambda \vartheta)} g(\vartheta) \\
& =\frac{1}{s} \int_{0}^{1} \mathrm{~d} \vartheta \frac{g(\vartheta)}{1+\frac{\lambda}{s} \vartheta}=\frac{1}{s} \bar{g}\left(\kappa=\frac{\lambda}{s}\right) .
\end{aligned}
$$

The scaling function $g(\vartheta)$ encoding the distribution $\mathcal{P}_{H}^{T}\left(t_{+}\right)$, and the scaling function $\bar{g}(\kappa)$ encoding its double Laplace transform $\tilde{P}(\lambda, s)$, are related by a simple integral transform which we denote $\mathcal{K}_{1}$,

$$
\mathcal{K}_{1}[g](\kappa):=\int_{0}^{1} \mathrm{~d} \vartheta \frac{g(\vartheta)}{1+\kappa \vartheta}=\bar{g}(\kappa) .
$$

For the case of interest, a fBm bridge of lenght $T$, this relation is more complicated since we can not compute directly the double Laplace transform of $\mathcal{P}_{H}^{\text {bridge }}\left(t_{+}\right)$, but only the transform of an unnormalised distribution, which we write $Z^{N}(T) \mathcal{P}_{H}^{\text {bridge }}\left(t_{+}\right)$. As we will see, the normalisation factor $Z^{N}(T)$, which is the probability density to return to 0 at time $T$, is a power law,

$$
Z^{N}(T)=C T^{\alpha-1},
$$

with some constant $C$. In this case, the double Laplace transform of the unnormalised distribution is computed as

$$
\begin{aligned}
\int_{0}^{\infty} \mathrm{d} T \int_{0}^{T} \mathrm{~d} t_{+} e^{-s T-\lambda t_{+}} C T^{\alpha-1} \mathcal{P}_{H}^{\text {bridge }}\left(t_{+}\right) \\
=\int_{0}^{1} \mathrm{~d} \vartheta \int_{0}^{\infty} \mathrm{d} T C T^{\alpha-1} e^{-T(s+\lambda \vartheta)} g(\vartheta) \\
=\frac{C \Gamma(\alpha)}{s^{\alpha}} \int_{0}^{1} \mathrm{~d} \vartheta \frac{g(\vartheta)}{\left(1+\frac{\lambda}{s} \vartheta\right)^{\alpha}} \\
\stackrel{!}{=} \frac{C \Gamma(\alpha)}{s^{\alpha}} \mathcal{K}_{\alpha}[g]\left(\kappa=\frac{\lambda}{s}\right)
\end{aligned}
$$

Here we generalised the $\mathcal{K}$ transform to another exponent,

$$
\mathcal{K}_{\alpha}[g](\kappa):=\int_{0}^{1} \mathrm{~d} \vartheta \frac{g(\vartheta)}{(1+\kappa \vartheta)^{\alpha}} .
$$

If $\bar{g}(\kappa)=\mathcal{K}_{\alpha}[g](\kappa)$ is the $\mathcal{K}_{\alpha}$ transform of a function $g(\vartheta)$ normalised to unity, then $\bar{g}(\kappa) \rightarrow 1$ for $\kappa \rightarrow 0$. If further $g(\vartheta)$ is time-reversal symmetric, $g(\vartheta)=g(1-\vartheta)$ for $\vartheta \in[0,1]$, then the function $\bar{g}(\kappa)$ has the symmetry

$$
\bar{g}(\kappa)=\frac{1}{(1+\kappa)^{\alpha}} \bar{g}\left(-\frac{\kappa}{1+\kappa}\right) .
$$

\section{FBm bridge with $H=\frac{1}{2}+\varepsilon$}

The path-integral approach presented in Section III yields an expression for the (unnormalised) density distribution of 
$t_{+}$for a bridge,

$$
\begin{aligned}
& Z^{\mathrm{pos}}\left(t_{+}, T\right)= \\
& \quad \int_{X_{0}=0}^{X_{T}=0} \mathcal{D}[X] \delta\left(\int_{0}^{T} \mathrm{~d} t \Theta\left(X_{t}\right)-t_{+}\right) e^{-S[X]} .
\end{aligned}
$$

It is useful to consider its double Laplace transform $(T \rightarrow s$ and $t_{+} \rightarrow \lambda$ ), which we denote with a tilde

$$
\begin{aligned}
& \tilde{Z}^{\mathrm{pos}}(\lambda, s)= \\
& \quad \int_{0}^{\infty} \mathrm{d} T e^{-s T} \int_{X_{0}=0}^{X_{T}=0} \mathcal{D}[X] e^{-S[X]-\lambda \int_{0}^{T} \mathrm{~d} t \Theta\left(X_{t}\right)} .
\end{aligned}
$$

Using the $\varepsilon$-expansion (16) for the action, we compute this perturbatively, expanding around Brownian motion. The resulting series in $\varepsilon$ has the form

$$
\tilde{Z}^{\mathrm{pos}}(\lambda, s)=\tilde{Z}_{0}^{\mathrm{pos}}(\lambda, s)+\varepsilon \tilde{Z}_{1}^{\mathrm{pos}}(\lambda, s)+\mathcal{O}\left(\varepsilon^{2}\right) .
$$

The first term of this expansion, the result for Brownian motion, is as in Eq. 47) obtained from the propagator $\tilde{W}^{+}$,

$$
\tilde{Z}_{0}^{\text {pos }}(\lambda, s)=\tilde{W}^{+}(\lambda, s)=\frac{1}{\sqrt{s}} \frac{1}{\sqrt{1+\kappa}+1}=\frac{\bar{g}_{0}(\kappa)}{2 \sqrt{s}} .
$$

Here we denoted

$$
\bar{g}_{0}(\kappa)=\int_{0}^{1} \mathrm{~d} \vartheta \frac{g_{0}(\vartheta)}{\sqrt{1+\kappa \vartheta}}=\frac{2}{\sqrt{1+\kappa}+1} .
$$

This can be inverted to

$$
g_{0}(\vartheta)=1
$$

This reproduces the known result that the probability distribution (2) for a Brownian bridge is uniform [30, 31].

To compute the order- $\varepsilon$ term $\tilde{Z}_{1}^{\text {pos }}(\lambda, s)$, we use the same diagrammatic rules as in Ref. [34], Section III D. These rules are easily expressed in Laplace variables, which is why we compute the expansion of $Z^{\text {pos }}(\lambda, s)$. The first order- $\varepsilon$ correction comes from the non-local interaction in the action, given in the second line of Eq. [16, and can be written as

$$
\tilde{Z}_{1 \mathrm{~A}}^{\mathrm{pos}}(\lambda, s)=2 \int_{0}^{\Lambda} \mathrm{d} y \int_{-\infty}^{\infty} \mathrm{d} x_{1} \int_{-\infty}^{\infty} \mathrm{d} x_{2} \tilde{W}^{+}\left(\lambda, s, 0, x_{1}\right) \partial_{x_{1}} \tilde{W}^{+}\left(\lambda, s+y, x_{1}, x_{2}\right) \partial_{x_{2}} \tilde{W}^{+}\left(\lambda, s, x_{2}, 0\right) .
$$

As explained in Ref. [34], the large- $y$ cutoff $\Lambda$, which is necessary as the integral is logarithmically divergent, is linked to the short-time (UV) regularisation $\tau$ introduced in Eq. (16) by $\Lambda=e^{-\gamma_{\mathrm{E}}} / \tau$. Performing the integrations over space variables and over $y$, and after some simplifications, we obtain

$$
\tilde{Z}_{1 \mathrm{~A}}^{\mathrm{pos}}(\lambda, s)=\frac{1}{\sqrt{s}}\left[\left(\frac{4}{\sqrt{\kappa+1}}+4\right) \ln (\sqrt{\kappa+1}+1)-\frac{2 \kappa+2+\sqrt{\kappa+1}}{\kappa} \ln (\kappa+1)+\frac{\ln (s \tau)+7-7 \ln (4)+\gamma_{\mathrm{E}}}{\sqrt{\kappa+1}+1}\right] .
$$

We have expressed the result in terms of the dimensionless variable $\kappa=\lambda / s$. The second order- $\varepsilon$ correction comes from the rescaling of the diffusive constant, c.f. Eq. (17). It is computed by rescaling $T$ in the result for the Brownian, setting $T \rightarrow D_{\varepsilon, \tau} T$. In Laplace variables, this is equivalent to writing

$$
\tilde{Z}_{0}^{\mathrm{pos}}(\lambda, s) \rightarrow \frac{1}{D_{\varepsilon, \tau}} \tilde{Z}_{0}^{\mathrm{pos}}\left(\frac{\lambda}{D_{\varepsilon, \tau}}, \frac{s}{D_{\varepsilon, \tau}}\right)
$$

Extracting the order- $\varepsilon$ term gives

$$
\tilde{Z}_{1 B}^{\text {pos }}(\lambda, s)=-\frac{1+\ln (\tau)}{2 \sqrt{s}} \frac{2}{\sqrt{1+\kappa}+1} .
$$

Resumming all order- $\varepsilon$ corrections,

$$
\tilde{Z}_{1}^{\text {pos }}(\lambda, s)=\tilde{Z}_{1 A}^{\text {pos }}(\lambda, s)+\tilde{Z}_{1 B}^{\text {pos }}(\lambda, s),
$$

the $\tau$ dependence vanishes. The $\ln (s)$ term in Eq. 63 is proportional to $\bar{g}_{0}(\kappa)$, such that we can recast it as an order- $\varepsilon$ correction to the exponent of the prefactor: $s^{-1 / 2} \rightarrow s^{H-1}+$
$\mathcal{O}\left(\varepsilon^{2}\right)$. This allows us to write the path integral (57) in the form

$$
\tilde{Z}^{\mathrm{pos}}(\lambda, s)=\frac{\Gamma(1-H)}{2 \sqrt{\pi} s^{1-H}}\left[\bar{g}_{0}(\kappa)+\varepsilon \bar{g}_{1}^{\mathrm{pos}}(\kappa)\right]+\mathcal{O}\left(\varepsilon^{2}\right) .
$$

With this choice of prefactor, the constant $C$ in Eq. (53) is $C=(2 \sqrt{\pi})^{-1}$, and $\bar{g}_{1}^{\text {pos }}(\kappa)$ is

$$
\begin{aligned}
\bar{g}_{1}^{\mathrm{pos}}(\kappa)= & 8\left(\frac{1}{\sqrt{\kappa+1}}+1\right) \ln (\sqrt{\kappa+1}+1) \\
& -2 \frac{2 \kappa+2+\sqrt{\kappa+1}}{\kappa} \ln (\kappa+1)+4 \frac{3-4 \ln (4)}{\sqrt{\kappa+1}+1} .
\end{aligned}
$$

We recall that this function contains contributions from $\tilde{Z}_{1 \mathrm{~A}}^{\text {pos }}$, $\tilde{Z}_{1 \mathrm{~B}}^{\text {pos }}$ and the expansion of $\frac{1}{\sqrt{\pi}} \Gamma\left(\frac{1}{2}-\varepsilon\right)=1+\varepsilon\left[\gamma_{\mathrm{E}}+\ln (4)\right]+$ $\mathcal{O}\left(\varepsilon^{2}\right)$, due to the choice of normalisation in Eq. 67).

We know that the distribution of the positive time has the form given in Eq. (49). After expanding it in $\varepsilon$ it gives

$$
\mathcal{P}_{H=\frac{1}{2}+\varepsilon}^{\text {bridge }}\left(t_{+}\right)=\frac{1}{T}\left[g_{0}(\vartheta)+\varepsilon g_{1}^{\text {pos }}(\vartheta)\right]+\mathcal{O}\left(\varepsilon^{2}\right),
$$


where, as before, $\vartheta=t_{+} / T$.

We have seen in Section IVC that the scaling functions $g(\vartheta)$ and $\bar{g}(\kappa)$ are related via the $\mathcal{K}_{1-H}$ transform, where the index of the transformation is fixed by the prefactor $s^{H-1}$ in Eq. 677.

Expanding w.r.t. $\varepsilon$ in the definition of the $\mathcal{K}$ transform gives

$$
\begin{aligned}
\bar{g}(\kappa) & =\int_{0}^{1} \mathrm{~d} \vartheta \frac{1}{(1+\kappa \vartheta)^{\frac{1}{2}-\varepsilon}} g(\vartheta) \\
& =\int_{0}^{1} \mathrm{~d} \vartheta \frac{1+\varepsilon \ln (1+\kappa \vartheta)}{\sqrt{1+\kappa \vartheta}}\left[g_{0}(\vartheta)+\varepsilon g_{1}(\vartheta)\right]+\mathcal{O}\left(\varepsilon^{2}\right) \\
& =\bar{g}_{0}(\kappa)+\varepsilon \int_{0}^{1} \mathrm{~d} \vartheta \frac{\left[g_{1}(\vartheta)+g_{0}(\vartheta) \ln (1+\kappa \vartheta)\right]}{\sqrt{1+\kappa \vartheta}} \\
& +\mathcal{O}\left(\varepsilon^{2}\right)
\end{aligned}
$$

The order- $\varepsilon$ correction $g_{1}(\vartheta)$ that we are looking for is then given by

$$
g_{1}(\vartheta)=\mathcal{K}_{\frac{1}{2}}^{-1}\left[\bar{g}_{1}(\kappa)-\bar{g}_{0,1}(\kappa)\right],
$$

where we have defined

$$
\begin{aligned}
\bar{g}_{0,1}(\kappa) & =\int_{0}^{1} \mathrm{~d} \vartheta \frac{\ln (1+\kappa \vartheta)}{\sqrt{1+\kappa \vartheta}} g_{0}(\vartheta) \\
& =\frac{2}{\kappa}\{2+\sqrt{1+\kappa}[\ln (\kappa+1)-2]\} .
\end{aligned}
$$

This contribution is valid both for $t_{+}$and $t_{\max }$, since both observables have the same distribution at order zero, and both have the same power law from scaling.

We now have to deal with the inverse $\mathcal{K}_{\frac{1}{2}}$ transform in Eq. (71). This is linked to the Abel transform, on which details are given in Appendix B The final result for the order- $\varepsilon$ correction is

$$
\begin{aligned}
g_{1}^{\mathrm{pos}}(\vartheta)=4[2 & -\frac{1}{\sqrt{\vartheta}+1}+\ln \left(\frac{\sqrt{\vartheta}+1}{4 \sqrt{\vartheta}}\right) \\
& \left.-\frac{1}{\sqrt{1-\vartheta}+1}+\ln \left(\frac{\sqrt{1-\vartheta}+1}{4 \sqrt{1-\vartheta}}\right)\right] .
\end{aligned}
$$

We can check that the integral of $g_{1}^{\text {pos }}(\vartheta)$ over $[0,1]$ vanishes, such that Eq. (69) is correctly normalised at order $\varepsilon$. We also checked that by computing numerically the $\mathcal{K}_{1 / 2}$ transform of this result reproduces $\bar{g}_{1}^{\text {pos }}(\kappa)-\bar{g}_{0,1}(\kappa)$ with excellent precision.

Close to the boundary, the asymptotics is

$$
g_{1}^{\text {pos }}(\vartheta) \underset{\vartheta \rightarrow 0,1}{\simeq}-2 \ln (\vartheta)-2 \ln (1-\vartheta) .
$$

This asymptotics can be recast into a power law consistent with scaling. The distribution of $t_{+}$for a $\mathrm{fBm}$ bridge with $H=\frac{1}{2}+\varepsilon$ can then be written as

$$
\mathcal{P}_{H=\frac{1}{2}+\varepsilon}^{\text {bridge }}\left(t_{+}\right)=\frac{\exp \left(\varepsilon\left[\mathcal{F}^{\operatorname{pos}}(\vartheta)-4\right]\right)}{T[\vartheta(1-\vartheta)]^{2 H-1}}+\mathcal{O}\left(\varepsilon^{2}\right) .
$$

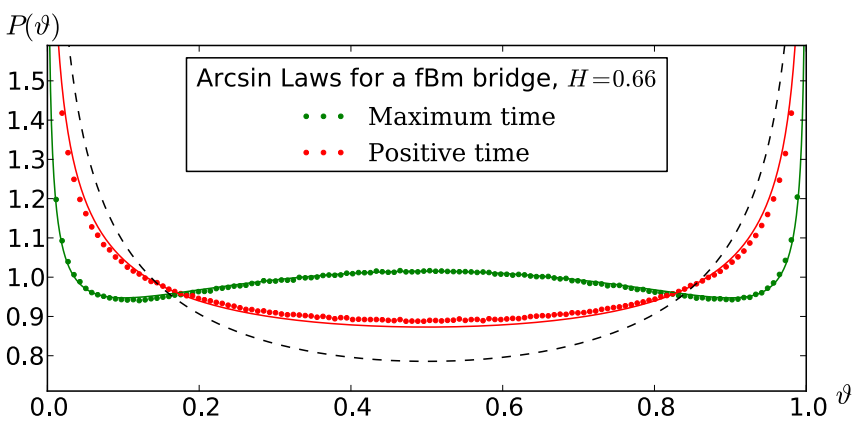

FIG. 4. Comparison of the two "Arcsine laws" for a fBm bridge with Hurst exponent $H=0.66$. Dots represent the distribution extracted from numerical simulations, the plain lines represent the analytical result at order $\varepsilon$ given in Eqs. (75) and (82, and the dashed line is the scaling form (identical for both observables).

The scaling function $\mathcal{F}^{\mathrm{pos}}(\vartheta)$ has by definition vanishing integral, and is given by

$$
\begin{aligned}
\mathcal{F}^{\mathrm{pos}}(\vartheta)=4[3 & -\frac{1}{\sqrt{\vartheta}+1}+\ln \left(\frac{\sqrt{\vartheta}+1}{4}\right) \\
& \left.-\frac{1}{\sqrt{1-\vartheta}+1}+\ln \left(\frac{\sqrt{\vartheta}+1}{4}\right)\right] .
\end{aligned}
$$

\section{E. Numerical results}

To test our analytical predictions, we compare them to results from numerical simulations. As in Ref. [34], we construct a large number of $\mathrm{fBm}$ paths using the Davis and Harte procedure, c.f. Ref. [27] for details on the numerical method. From these samples, we construct a numerical estimation $\mathcal{P}_{H}^{\text {bridge }}\left(t_{+}\right)$of the distribution of $t_{+}$for various values of $H$, choosing $T=1$. This is shown on Fig. 4, where results for the distributions of both $t_{+}$and $t_{\max }$ are given. To compare to the analytical result $(76)$, we extract $\mathcal{F}_{\text {num }}^{\text {pos }}$ from these distributions, using

$$
\mathcal{F}_{\text {num }}^{\text {pos }}(\vartheta)=\frac{1}{\varepsilon} \ln \left(T[\vartheta(1-\vartheta)]^{2 H-1} \mathcal{P}_{H=\frac{1}{2}+\varepsilon}^{\text {briige }}(\vartheta)\right) .
$$

As is shown in Fig. 5 (left), when $\varepsilon \rightarrow 0, \mathcal{F}_{\text {num }}^{\text {pos }}(\vartheta)$ converges to $\mathcal{F}^{\text {pos }}(\vartheta)$. The deviation being antisymmetric in $\varepsilon$ strongly suggests that there is an order- $\varepsilon^{2}$ correction to the distribution of $t_{+}$, which we did not calculate here.

\section{EXTREMUM OF FBM BRIDGES}

In Ref. [34], a general formula was derived for the path integral over fBm paths $X_{t}$ starting at $m_{1}$, going to $x_{0} \approx 0$ at time $t_{1}$ and ending in $m_{2}$ at time $t_{1}+t_{2}=T$, while staying positive, $X_{t}>0$ for all $t \in[0, T]$. This quantity, denoted $Z^{+}\left(m_{1}, t_{1} ; x_{0} ; m_{2}, t_{2}\right)$, is the first-order term in an $\varepsilon$ expansion. It was used [34] to derived results about extremal properties of $\mathrm{fBm}$ in the unconstrained case: Both the distribution 

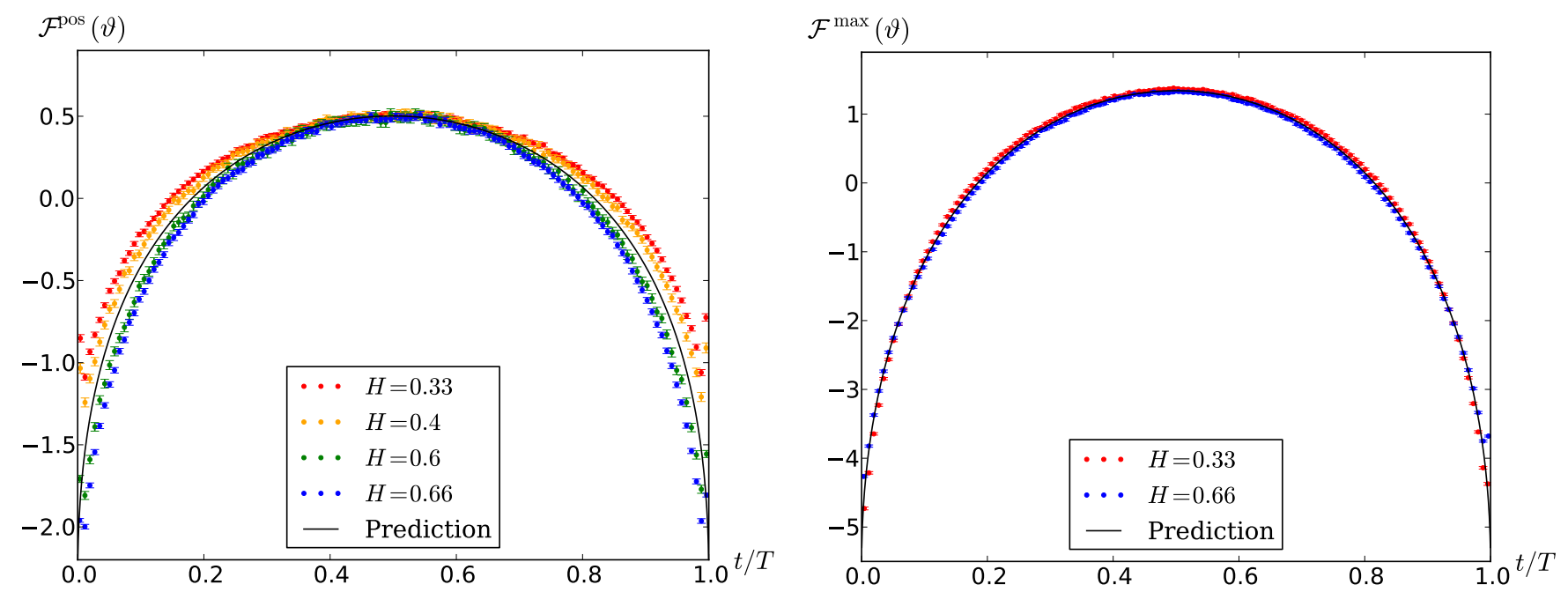

FIG. 5. Left: Numerical estimation of the scaling function $\mathcal{F}^{\text {pos }}(\vartheta)$, from top to bottom for $H=0.33$ (red dots), $H=0.4$ (orange dots), $H=0.6$ (green dots), and $H=0.66$ (blue dots), compared to the analytical result given in Eq. 76 (plane line). Right: $i b i d$ for $\mathcal{F}^{\text {max }}(\vartheta)$ for $H=0.33$ (blue dots, bottom) and $H=0.66$ (red dots, top), the analytical result (plane line) is given in Eq. (83). For both plots, and for each value of $H$, the statistics is done with $5 \times 10^{6}$ sampled paths, discretized with $N=2^{12}$ points.

of the maximum $m$, and the time $t_{\max }$ when this maximum is achieved, were computed, as well as their joint distribution. Comparison to numerical simulations showed that this result is of great precision for small $\varepsilon$, and of good precision for larger vales of $\varepsilon$.

Here we apply these results to $\mathrm{fBm}$ bridges. The general result for $Z^{+}\left(m_{1}, t_{1} ; x_{0} ; m_{2}, t_{2}\right)$, restricted to $m_{1}=m_{2}=m$, and choosing $t_{1}+t_{2}=T$, immediately gives the joint distribution of the maximum $m$, and the time $t_{\max }=t_{1}$ when this maximum is attained. In a second step, we can then integrate over $t_{1}$ at $T$ fixed, or over $m$ at $t_{1}$ and $t_{2}$ fixed, to obtain the distributions of $m$ and $t_{\max }$.

We will finally rederive these results in a simpler way, taking advantage of the scaling transformations introduced in section IVC

\section{A. Distribution of the time to reach the maximum}

Starting with Eq. (44) of Ref. [34] and following the procedure in its section IV.C, we express the probability for $t_{\max }$, denoted $\mathcal{P}_{H}^{\text {bridge }}\left(t_{\max }\right)$, as

$$
\mathcal{P}_{H}^{\text {bridge }}\left(t_{\max }\right)=\frac{1}{Z^{N}(T)} \int_{0}^{\infty} \mathrm{d} m Z^{+}\left(m, t ; x_{0} ; m, T-t\right) .
$$

The integral over $m$ accounts for all possible values of the maximum. $Z^{N}(T)$ is a normalisation factor such that the integral over $t_{\max }$ of $\mathcal{P}_{H}^{\text {bridge }}\left(t_{\max }\right)$ is normalised to unity,

$$
\begin{aligned}
Z^{N}(T) & =\int_{0}^{T} \mathrm{~d} t \int_{0}^{\infty} \mathrm{d} m Z^{+}\left(m, t ; x_{0} ; m, T-t\right) \\
& =\frac{x_{0}^{2-4 \varepsilon}}{\sqrt{4 \pi}}\left(1+\varepsilon C_{1}\right)+\mathcal{O}\left(\varepsilon^{2}\right)
\end{aligned}
$$

The constant $C_{1}$ can be computed from $Z^{+}$, but it is equivalent to require that the order- $\varepsilon$ term in Eq. (78) does not change the normalisation, such that the distribution $\mathcal{P}_{H}^{\text {bridge }}\left(t_{\max }\right)$ remains normalised to one.

Expanding the distribution of $t_{\max }$ in the same way as for Eq. [69], the order- $\varepsilon$ term becomes, setting again $\vartheta=$ $t_{\max } / T$, and $T=1$

$$
\begin{aligned}
g_{1}^{\max }(\vartheta)= & 2 \sqrt{\pi} \int_{0}^{\infty} \mathrm{d} m\left[Z_{1}^{+}\left(m, \vartheta ; x_{0} ; m, 1-\vartheta\right)\right. \\
& \left.-C_{1} Z_{0}^{+}\left(m, \vartheta ; x_{0} ; m, 1-\vartheta\right)\right] \\
=2[ & 6(\sqrt{1-\vartheta}+\sqrt{\vartheta})-3 \vartheta \ln (1-\vartheta)-3(1-\vartheta) \ln (\vartheta) \\
+ & (4-3 \vartheta) \ln (2-\vartheta)+(3 \vartheta+1) \ln (\vartheta+1) \\
+ & (6 \vartheta-4) \operatorname{arcth}(\sqrt{1-\vartheta})+(2-6 \vartheta) \operatorname{arcth}(\sqrt{\vartheta}) \\
- & 8-4 \ln (2)] .
\end{aligned}
$$

This result will be checked from Eq. (107) given below. Demanding that $g_{1}^{\max }(\vartheta)$ has integral zero fixed the constant $C_{1}$ to $C_{1}=4 \ln (2)-\gamma_{\mathrm{E}}$.

Close to the boundary, the correction has the same asymptotics as in the calculation for $t_{+}$, namely

$$
g_{1}^{\max }(\vartheta) \underset{\vartheta \rightarrow 0,1}{\simeq}-2 \ln (\vartheta)-2 \ln (1-\vartheta)
$$

which indicates the same change in the power-law behaviour of $\mathcal{P}_{H}^{\text {bridge }}\left(t_{\max }\right)$. Again taking an exponential resummation of the order- $\varepsilon$ correction, we obtain a formula similar to Eq. 75, but with a different scaling function $\mathcal{F}^{\max }(\vartheta)$,

$$
\mathcal{P}_{H=\frac{1}{2}+\varepsilon}^{\text {bridge }}\left(t_{\text {max }}\right)=\frac{\exp \left(\varepsilon\left[\mathcal{F}^{\max }(\vartheta)-4\right]\right)}{T[\vartheta(1-\vartheta)]^{2 H-1}}+\mathcal{O}\left(\varepsilon^{2}\right) .
$$


$\mathcal{F}^{\max }(\vartheta)$ is a bounded function of $\vartheta \in[0,1]$ and can be expressed from Eq. 80 , as

$$
\mathcal{F}^{\max }(\vartheta)=g_{1}^{\max }(\vartheta)+2 \ln (\vartheta(1-\vartheta))+4 .
$$

The constant 4 was added in Eq. (83) and subtracted in Eq. (82) to have $\int_{0}^{1} \mathrm{~d} \vartheta g_{1}^{\max }(\vartheta)=\int_{0}^{1} \mathrm{~d} \vartheta \mathcal{F}^{\max }(\vartheta)=0$.

The two distributions, for $t_{+}$and $t_{\max }$, at order $\varepsilon$ are plotted in Fig. 4. While both functions have the same power-law behavior for $\vartheta$ close to 0 or 1 , their difference is clearly visible. The result 83 for $\mathcal{F}^{\max }(\vartheta)$ is compared with great precision to numerical simulations on figure 5 (right).

\section{B. The maximum-value distribution}

Similarly to the distribution of $t_{\max }$, the distribution of the maximum value $m=\max _{t \in[0, T]} X_{t}$ can be expressed from the result for $Z^{+}$given in Eq. (44) of Ref. [34]:

$$
\mathcal{P}_{H}^{\text {bridge }}(m)=\frac{1}{Z^{N}(T)} \int_{0}^{T} \mathrm{~d} t Z^{+}\left(m, t ; x_{0} ; m, T-t\right) .
$$

This calculation is rather cumbersome, but it is possible to give a simpler derivation, where we do not constrain paths to go close to the boundary, but construct $\mathcal{P}_{H}^{\text {bridge }}(m)$ by taking a derivative of its cumulative distribution, the survival probability, conditioned such that the end point of the process is the same as the starting point. In this framework, the order- $\varepsilon$ correction to $\mathcal{P}_{H}^{\text {bridge }}(m)$ can, due to the non-local term in the action (16), be expressed in Laplace variables $(T \rightarrow s)$ using the diagrammatic rules of Ref. [34]. The integrals to be computed are

$$
\begin{aligned}
\tilde{Z}_{1 A}^{\max }(m, s) & =2 \partial_{m} \int_{0}^{\Lambda} \mathrm{d} y \int_{x_{1}, x_{2}>0} \tilde{P}_{0}^{+}\left(m, x_{1} ; s\right) \partial_{x_{1}} \tilde{P}_{0}^{+}\left(x_{1}, x_{2} ; s+y\right) \partial_{x_{2}} \tilde{P}_{0}^{+}\left(x_{2}, m ; s\right) \\
& =2(a+1) e^{2 a} \operatorname{Ei}(-4 a)-2 \operatorname{Ei}(-2 a)+2 e^{-2 a}\left\{a\left[\ln \left(\frac{m^{2}}{4 \tau}\right)-\ln (a)-1\right]+\ln \left(\frac{2 \tau}{m^{2}}\right)-\gamma_{\mathrm{E}}\right\},
\end{aligned}
$$

where $a:=\sqrt{s} m$ is a dimensionless variable, $\Lambda=e^{-\gamma_{\mathrm{E}}} / \tau$, and the propagator $\tilde{P}_{0}^{+}\left(x_{1}, x_{2} ; s\right)$ is defined in Eq. (39). To deal with the inverse Laplace transform, we use formulas (G10) and (G11) derived in Ref. [34], plus similar formulas collected in appendix C. The final result for the correction after the inverse Laplace transformation is

$$
Z_{1 A}^{\max }(m, T)=\frac{z e^{-z^{2}}}{\sqrt{\pi} T}\left\{2 z \sqrt{\pi} e^{z^{2}} \operatorname{erfc}(z)+4\left(1-z^{2}\right) \mathcal{J}\left(z^{2}\right)+2 z^{2}\left[\ln \left(\frac{T z^{2}}{\tau}\right)+\gamma_{\mathrm{E}}-1\right]+\ln \left(\frac{\tau^{3}}{T^{3} z^{8}}\right)-4 \gamma_{\mathrm{E}}+1\right\}
$$

We introduced the scaling variable $z:=m / \sqrt{T}$. The special function $\mathcal{J}$ defined in Ref. [34] is

$$
\mathcal{J}(x)=\frac{1}{2} \pi \operatorname{erfi}(\sqrt{x})-x_{2} F_{2}\left(1,1 ; \frac{3}{2}, 2 ; x\right) .
$$

For a Brownian bridge we have

$$
Z_{0}^{\max }(m, T)=\frac{m}{\sqrt{\pi} T^{\frac{3}{2}}} e^{-\frac{m^{2}}{T}},
$$

which, after normalisation, allows to recover the distribution (4).

The second order- $\varepsilon$ correction, which comes from the rescaling of the diffusive constant, is obtained by replacing $T \rightarrow D_{\varepsilon, \tau} T$ in Eq. 88); for the order- $\varepsilon$ term this gives

$$
Z_{1 B}^{\max }(m, T)=\frac{z e^{-z^{2}}}{\sqrt{\pi} T}\left(2 z^{2}-3\right)(1+\ln \tau) .
$$

Resumming these corrections up to order $\varepsilon$ cancels all $\tau$ dependencies; recasting the relevant corrections into the powerlaw prefactor and the Gaussian tail and expressing the result in terms of the dimensionless variable $y:=m / T^{H}$ finally yields

$$
\begin{aligned}
\mathcal{P}_{H}^{\text {bridge }}(m) & =2 \sqrt{\pi} T^{H}\left[Z_{0}^{\max }+\varepsilon\left(Z_{1 A}^{\max }+Z_{1 B}^{\max }\right)\right]+\mathcal{O}\left(\varepsilon^{2}\right) \\
& =\frac{2 y^{1-8 \varepsilon}}{T^{H}} e^{-y^{2} A_{\varepsilon}+\varepsilon \mathcal{G}(y)+\mathrm{cst}}+\mathcal{O}\left(\varepsilon^{2}\right) .
\end{aligned}
$$

The special function $\mathcal{G}$ appearing here is as defined in Refs. [32-34],

$$
\begin{aligned}
\mathcal{G}(y)= & -4\left(y^{2}-1\right) \mathcal{J}\left(y^{2}\right)+2 \sqrt{\pi} e^{y^{2}} y \operatorname{erfc}(y) \\
& +2 y^{2}\left[\ln \left(4 y^{2}\right)+\gamma_{\mathrm{E}}\right]-4 \gamma_{\mathrm{E}}-2 .
\end{aligned}
$$

This result contains several non-trivial predictions: First, at small $m$, the distribution $\mathcal{P}_{H}^{\text {bridge }}(m)$ has a power law given by $m^{1-8 \varepsilon+\mathcal{O}\left(\varepsilon^{2}\right)}$. This can be obtained by considering the probability starting at $m$ to remain positive (survive) up to time $T$,

$$
\mathcal{S}(T, m):=\int_{0}^{m} \mathrm{~d} m_{1} \mathcal{P}_{H}\left(m_{1}\right) .
$$

In this relation the dependence of $\mathcal{P}_{H}(m)$ on $T$ is implicit. It is valid both for the case of a bridge and of a free endpoint. To 

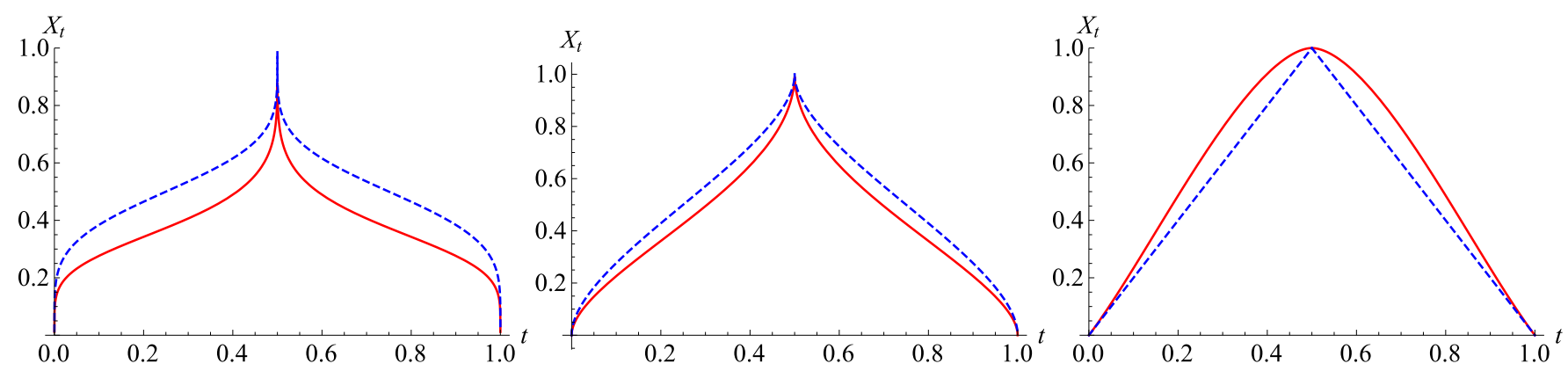

FIG. 6. Plain red line: optimal paths for fBm conditioned to $X_{0}=0, X_{1 / 2}=1$ and $X_{1}=0$, for, from left to right, $H=0.1, H=0.25$ and $H=1$. The blue dashed-line represents the optimal paths when neglecting the correlation between $[0,1 / 2]$ and $[1 / 2,1]$.

survive in a bridge in the limit of $m \rightarrow 0$ demands to survive both in the beginning and at the end, thus we expect that for small $m$

$$
\mathcal{S}^{\text {bridge }}(T, m) \sim\left[\mathcal{S}^{\text {free }}(T, m)\right]^{2} .
$$

Using the result of Ref. [34] that $\mathcal{P}_{H}^{\text {free }}(m) \sim m^{\frac{1}{H}-2}$ implies that

$$
\mathcal{P}_{H}^{\text {bridge }}(m) \sim m^{\frac{2}{H}-3} .
$$

This is in agreement with our order- $\varepsilon$ result.

Second, at large $m, \mathcal{P}_{H}^{\text {bridge }}(m)$ has a Gaussian tail with the dimensionless variable $y^{2}=z^{2} / T^{2 \varepsilon}=m^{2} / T^{2 H}$ and a non-trivial number $A_{\varepsilon}=1+4 \varepsilon \ln (2)+\mathcal{O}\left(\varepsilon^{2}\right)$. We will see in the next section why this number appears, and how we can compute it exactly (i.e. for all $H$ ).

Third, there is a crossover in the power-law behavior at large $y$, given by the asymptotic behaviour of the function $\mathcal{G}(y)$

$$
\mathcal{G}(y) \underset{y \rightarrow \infty}{\simeq} 4 \ln (y) .
$$

This yields a subleading power-law behaviour at large $m$

$$
\mathcal{P}_{H}^{\text {bridge }}(m) e^{A_{\varepsilon} \frac{m^{2}}{T^{2 H}}} \sim m^{1-4 \varepsilon+\mathcal{O}\left(\varepsilon^{2}\right)} .
$$

\section{Optimal path for $\mathrm{fBm}$, and the tail of the maximum distribution}

In this section, we study the tail of the maximum distribution for $\mathrm{fBm}$. Contrary to a process with a free endpoint, the maximum is not taken at the end, and as a consequence the tail is not simply given by the known propagator evaluated at time $T$ at position $m$.

We start with some general considerations: If we choose $t_{1}, \ldots, t_{n} \in \mathbb{R}$, then the density distribution for a $\mathrm{fBm}$ path $X_{t}$ to take values $X_{t_{1}}=x_{1}, \ldots, X_{t_{n}}=x_{n}$ can be expressed, using the Gaussian nature of the process $X_{t}$, as

$$
\mathcal{P}_{n}\left(x_{1}, x_{2}, \ldots, x_{n}\right)=\exp \left(-\frac{1}{2} \sum_{i j} x_{i} \mathcal{M}_{i j} x_{j}\right) .
$$

The matrice $\mathcal{M}_{i j}$ is given by

$$
\mathcal{M}_{i j}^{-1}=\left\langle X_{t_{i}} X_{t_{j}}\right\rangle=t_{i}^{2 H}+t_{j}^{2 H}-\left|t_{i}-t_{j}\right|^{2 H} .
$$

To study bridges, consider now two points, $x_{1}=x$ at time $t_{1}=t$ with $0<t<T$ and $x_{2}=0$ at time $t_{2}=T$. The probability distribution of $x$ given $x_{T}=0$ is then given by

$$
\mathcal{P}\left(x_{t}=x \mid x_{T}=0\right)=\mathcal{P}_{2}(x, 0)=\exp \left(-\frac{\mathcal{M}_{11} x^{2}}{2}\right) .
$$

The matrix element in question is (with $\vartheta=t / T$ )

$$
\frac{\mathcal{M}_{11}}{2}=\frac{1}{T^{2 H}} \frac{1}{4 \vartheta^{2 H}-\left[\vartheta^{2 H}-(1-\vartheta)^{2 H}+1\right]^{2}} .
$$

It takes its minimum for $\vartheta=\frac{1}{2}$. The tail for the maximum of a bridge is thus given by Eq. 999 with the matrix element $\mathcal{M}_{11}$ in Eq. 100 evaluated at $\vartheta=\frac{1}{2}$ :

$$
\begin{aligned}
\mathcal{P}_{T}(m) & \approx \mathcal{P}\left(x_{T / 2}=m \mid x_{T}=0\right) \\
& =e^{-\frac{m^{2}}{T^{2 H}} \frac{4^{H}}{4-4^{H}}+\mathcal{O}(\ln (m))} .
\end{aligned}
$$

This heuristic argument is consistent with the result from our $\varepsilon$ expansion, and allows us to predict the exact value of the constant $A_{\varepsilon}$,

$$
A_{\varepsilon}=\frac{4^{H}}{4-4^{H}}=1+4 \ln (2) \varepsilon+\mathcal{O}\left(\varepsilon^{2}\right) .
$$

We can go further and study the shape of the optimal path with conditions $X_{0}=X_{1}=0$ and $X_{1 / 2}=1$. This is done by considering $\mathcal{P}_{n}(x, 1,0)$, taken at time $t_{1}=t, t_{2}=1 / 2$ and $t_{3}=T=1$. We then find $X_{t}^{\mathrm{SP}}=x$ which minimises the "energy" $-\ln \mathcal{P}_{3}(x, m, 0)$. This is for $0 \leq \vartheta \leq \frac{1}{2}$ achieved for

$X_{t}^{\mathrm{SP}}=\frac{m}{4-4^{H}}\left[2-2(1-2 \vartheta)^{2 H}+4^{H}(1-\vartheta)^{2 H}+4^{H} \vartheta^{2 H}-4^{H}\right]$.

For $\frac{T}{2}<t \leq T$ one has $X_{t}^{\mathrm{SP}}=X_{T-t}^{\mathrm{SP}}$. This is represented for $m=1$ and $T=1$ in red in Fig. 6 for various values of $H$. It is interesting to observe that this optimal path is not a straight line going from $X_{0}=0$ to $X_{1 / 2}=1$ and back to $X_{1}=1$, but at $t=1 / 2$ peaked for $H<1 / 2$, and smoothened for 
$H>1 / 2$. It is equivalently interesting to compare this to the optimal path which goes from $X_{0}=0$ to $X_{1 / 2}=1$, without imposing any constraint at $t=1$, plus a similar segment from $X_{1 / 2}=1$ to $X_{1}=0$ without constraint on $X_{0}$ (blue dashed lines). This would indeed be the optimal path if there were no correlations between times $t<1 / 2$ and $t>1 / 2$.

We finally note that the limit of $H \rightarrow 1$ is non-trivial, and given by (see right of Fig. 6)

$$
\begin{aligned}
X_{t}^{\mathrm{SP}}= & \frac{m}{\ln (4)}\left\{(1-2 \vartheta)^{2} \ln (1-2 \vartheta)-2(1-\vartheta)^{2} \ln (1-\vartheta)\right. \\
& +\vartheta[\ln (16)-2 \vartheta \ln (4 \vartheta)]\}, \quad 0 \leq t \leq \frac{T}{2} \quad \text { (104) }
\end{aligned}
$$

and $X_{t}^{\mathrm{SP}}=X_{T-t}^{\mathrm{SP}}$ for $\frac{T}{2}<t<T$. We expect this also to be the lowest-energy fluctuation for the $\mathrm{fBm}$ bridge.

\section{Joint Distribution of $m$ and $t_{\max }$}

To obtain the joint distribution of $m$ and $t_{\max }$, we start with Eq. (44) of Ref. [34], and specify $m_{1}=m_{2}=m$. This is equivalent, in the notations of [34], to setting

$$
y_{1}=\frac{m}{\sqrt{2} \vartheta^{H}}, y_{2}=\frac{m}{\sqrt{2}(1-\vartheta)^{H}} \text { where } \vartheta=\frac{t_{\max }}{T} .
$$

The resulting expression can more compactly be written in terms of

$$
v:=\frac{m}{\sqrt{2}[\vartheta(1-\vartheta)]^{H}} .
$$

Recasting terms proportional to $\ln (\vartheta), \ln (1-\vartheta)$ and $\ln (v)$ into the prefactor, we get

$$
\begin{aligned}
& Z^{+}\left(m, \vartheta ; x_{0} ; m, 1-\vartheta\right) \\
& =\frac{x_{0}^{2-4 \varepsilon} v^{2-8 \varepsilon} e^{-\frac{v^{2}}{2}}}{2 \pi[\vartheta(1-\vartheta)]^{3 H-1}}\left\{1+\varepsilon\left[\mathcal{F}(v, \vartheta)+C_{2}\right]\right\}+\mathcal{O}\left(\varepsilon^{2}\right)
\end{aligned}
$$

with

$$
\begin{aligned}
\mathcal{F}(v, \vartheta)= & \frac{\mathcal{I}(v(1-\vartheta))+\mathcal{I}(v \vartheta)-\mathcal{I}(v)+2\left(v^{2}-1\right)}{v^{2} \vartheta(1-\vartheta)} \\
& -\frac{\mathcal{I}(v(1-\vartheta))}{1-\vartheta}-\frac{\mathcal{I}(v \vartheta)}{\vartheta}+2 \mathcal{I}(v \sqrt{1-\vartheta}) \\
& +2 \mathcal{I}(v \sqrt{\vartheta})+v^{2}\left(\ln \left(2 v^{2}\right)+\gamma_{\mathrm{E}}\right) \\
& -12-8 \ln (2), \\
C_{2}= & 4\left[2-\gamma_{\mathrm{E}}+\ln (2)\right]
\end{aligned}
$$

First, this result allows us to recover Eqs. (80) and 83), noting that

$$
\mathcal{F}^{\max }(\vartheta)=\sqrt{\frac{2}{\pi}} \int_{0}^{\infty} \mathrm{d} v v^{2} e^{-\frac{v^{2}}{2}} \mathcal{F}(v, \vartheta)
$$

As we defined $\int_{0}^{1} \mathrm{~d} \vartheta \mathcal{F}^{\max }(\vartheta)=0$, there is an additional constant $C_{2}$, related to the prefactor $v^{-8 \varepsilon}$ in Eq. 107).
Second, we can extract the conditional probability of $v$, given $\vartheta$. This is interesting since for a Brownian the latter depends only on the variable $v$ introduced in Eq. (106),

$$
\mathcal{P}_{H=\frac{1}{2}}^{\text {bridge }}(v \mid \vartheta)=\sqrt{\frac{2}{\pi}} v^{2} e^{-\frac{v^{2}}{2}} .
$$

For a generic value of $H=\frac{1}{2}+\varepsilon$, our $\varepsilon$ expansion, recast in an exponential form, gives

$$
\begin{aligned}
\mathcal{P}_{H}^{\text {bridge }}(v \mid \vartheta)= & \sqrt{\frac{2}{\pi}} v^{\frac{2}{H}-2} e^{-\frac{v^{2}}{2}+\varepsilon\left[\mathcal{F}(v, \vartheta)+C_{2}-\mathcal{F}^{\max }(\vartheta)\right]} \\
& +\mathcal{O}\left(\varepsilon^{2}\right) .
\end{aligned}
$$

The functions $\mathcal{F}(v, \vartheta)$ and $\mathcal{F}^{\max }(\vartheta)$ are defined in Eqs. 108 and (83). The exponent in Eq. (112) can be derived from scaling. To this aim, note that the probability to have a maximum of $m$ up to time $T$ is

$$
\mathcal{P}_{H}(m)=\partial_{m} \mathcal{S}(T, m) .
$$

On the other hand, the probability that the maximum $m$ is taken at time $T$ is

$$
\mathcal{P}_{H}(m \mid T)=\partial_{T} \mathcal{S}(T, m) .
$$

We conclude that for small $m$

$$
\mathcal{P}_{H}^{\text {bridge }}(m \mid T) \sim \frac{m}{T} \mathcal{P}_{H}^{\text {bridge }}(m) \sim m^{\frac{2}{H}-2} \sim v^{\frac{2}{H}-2} .
$$

This exponent, written in Eq. 112, agrees with the perturbative expansion

$$
\frac{2}{H}-2=2-8 \varepsilon+\mathcal{O}\left(\varepsilon^{2}\right) .
$$

Finally, using the result $[100$, and expressing it in terms of $v$ predicts a tail $e^{-A_{\varepsilon}^{\prime} v^{2}}$, with

$$
\begin{aligned}
A_{\varepsilon}^{\prime} & =\frac{2[\vartheta(1-\vartheta)]^{2 H}}{4 \vartheta^{2 H}-\left[\vartheta^{2 H}-(1-\vartheta)^{2 H}+1\right]^{2}} \\
& =\frac{1}{2}\left[1+\varepsilon^{2} \frac{[(1-\vartheta) \ln (1-\vartheta)+\vartheta \ln (\vartheta)]^{2}}{2(1-\vartheta) \vartheta}+\mathcal{O}\left(\varepsilon^{3}\right)\right] .
\end{aligned}
$$

Thus our resummation (112) is correct to order $\varepsilon$; whether at higher order it is preferential to use $v$ introduced in Eq. (106) with $A_{\varepsilon}^{\prime}$ given in Eq. (117), or whether one should keep $e^{-v^{2} / 2}$ for the tail and redefine $v$ can only be answered after a second-order calculation.

We verified the prediction 112 for $\mathcal{P}_{H}^{\text {bridge }}(v \mid \vartheta)$ numerically, see Fig. 7. The agreement is good for $H$ close to $\frac{1}{2}$, both for $\varepsilon=-\frac{1}{10}$ and $\varepsilon=\frac{1}{10}$ (left two figures). Corrections of or$\operatorname{der} \varepsilon^{2}$ can be anticipated, since our numerical results for both $\varepsilon=-\frac{1}{10}$ and $\varepsilon=\frac{1}{10}$ show approximately the same (small) deviation from the analytics, independent of the sign of $\varepsilon$.

These putative $\mathcal{O}\left(\varepsilon^{2}\right)$ corrections also explain the larger systematic deviations for $H=\frac{2}{3}$, i.e. $\varepsilon=\frac{1}{6}$ (right plot). 

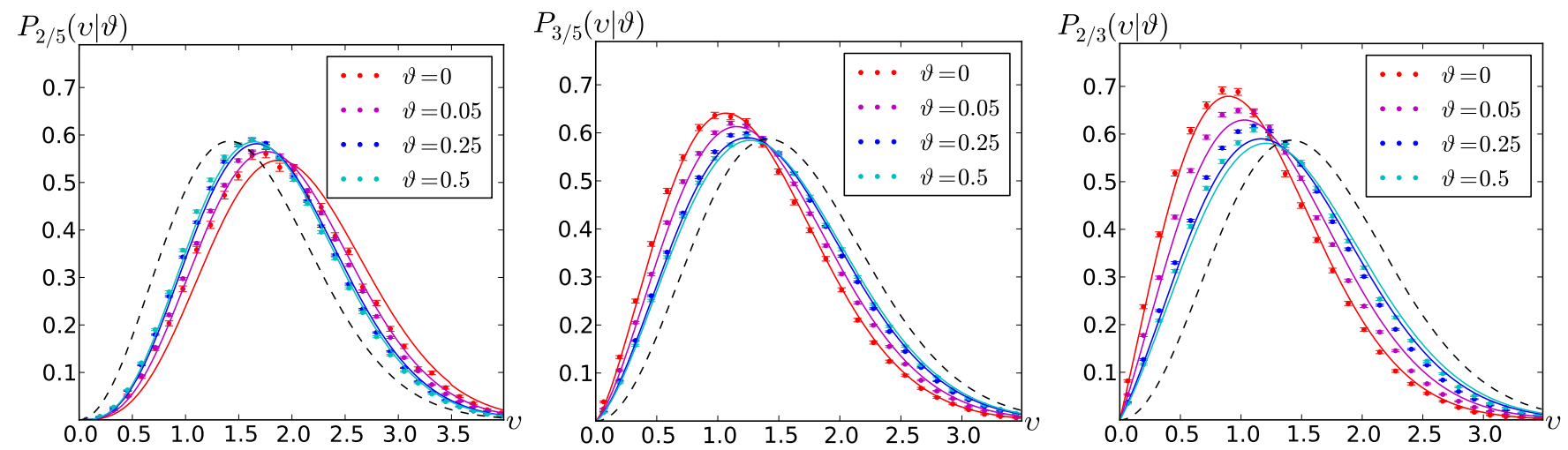

FIG. 7. Numerical results for $P_{H}(v \mid \vartheta)$ for $H=\frac{2}{5}$ (left), $H=\frac{3}{5}$ (middle) and $H=\frac{2}{3}$ (right). The values of $\vartheta$ are chosen as $\vartheta=0, \vartheta=0.05$, $\vartheta=0.25$ to $\vartheta=0.5$, the maximum useful value due to the symmetry $\vartheta \rightarrow 1-\vartheta$. We used $N=2^{18}$ points, and $5 \times 10^{6}$ samples.

\section{CONCLUSIONS}

In this article we developed a systematic analytical framework to treat bridge processes for fractional Brownian motion, in an expansion around Brownian motion. We considered the probability of the time $t_{+}$that a bridge process is positive, and of the time $t_{\max }$ it achieves its maximum. For a Brownian bridge, both $t_{+}$and $t_{\max }$ have the same uniform probability distribution. For a fractional Brownian bridge, both observables have the same power-law behavior for times close to the beginning and end, but the subleading scaling functions are rather different. We calculate them to first order in $\varepsilon$, and verified them to high precision with numerical simulations. We also obtained and checked the joint distribution of the maximum $m$, and the time $t_{\max }$ when this maximum is taken. These tests were possible due to the development of an efficient algorithm to generate samples of fBm bridges.

\section{ACKNOWLEDGMENTS}

We thank P. Krapivsky, K. Mallick, A. Rosso and T. Sadhu for stimulating discussions, and PSL for support through grant ANR-10-IDEX-0001-02-PSL.

\section{Appendix A: Details on correlation functions for the bridge}

Starting from Eqs. (6) and (7), and inserting the identity $\delta(x)=\int_{-\infty}^{\infty} e^{i k x} \frac{\mathrm{d} k}{2 \pi}$, we obtain

$$
\begin{aligned}
\left\langle\delta\left(X_{T}-a\right)\right\rangle & =\int_{-\infty}^{\infty} \frac{\mathrm{d} k}{2 \pi}\left\langle e^{i k\left(X_{T}-a\right)}\right\rangle \\
& =\int_{-\infty}^{\infty} \frac{\mathrm{d} k}{2 \pi} e^{-i k a} e^{-\frac{k^{2}}{2}\left\langle X_{T}^{2}\right\rangle} \\
& =\frac{e^{-\frac{a^{2}}{2\left\langle X_{T}^{2}\right\rangle}}}{\sqrt{2 \pi} \sqrt{\left\langle X_{T}^{2}\right\rangle}}
\end{aligned}
$$

$$
\begin{aligned}
\left\langle X_{t_{1}} \delta\left(X_{T}-a\right)\right\rangle & =\int_{-\infty}^{\infty} \frac{\mathrm{d} k}{2 \pi}\left\langle X_{t_{1}} e^{i k\left(X_{T}-a\right)}\right\rangle \\
& =\int_{-\infty}^{\infty} \frac{\mathrm{d} k}{2 \pi} e^{-i k a} i k\left\langle X_{t_{1}} X_{T}\right\rangle e^{-\frac{k^{2}}{2}\left\langle X_{T}^{2}\right\rangle} \\
& =\frac{e^{-\frac{a^{2}}{2\left\langle X_{T}^{2}\right\rangle}}}{\sqrt{2 \pi} \sqrt{\left\langle X_{T}^{2}\right\rangle}} \frac{a\left\langle X_{t_{1}} X_{T}\right\rangle}{\left\langle X_{T}^{2}\right\rangle}, \\
\left\langle X_{t_{1}}\right. & \left.X_{t_{2}} \delta\left(X_{T}-a\right)\right\rangle=\int_{-\infty}^{\infty} \frac{\mathrm{d} k}{2 \pi}\left\langle X_{t_{1}} X_{t_{2}} e^{i k\left(X_{T}-a\right)}\right\rangle \\
= & \int_{-\infty}^{\infty} \frac{\mathrm{d} k}{2 \pi} e^{-i k a} e^{-\frac{k^{2}}{2}\left\langle X_{T}^{2}\right\rangle} \\
& \times\left[\left\langle X_{t_{1}} X_{t_{2}}\right\rangle\right. \\
= & \frac{\left.e^{2}\left\langle X_{t_{1}} X_{T}\right\rangle\left\langle X_{t_{2}} X_{T}\right\rangle\right]}{\sqrt{2 \pi} \sqrt{\left\langle X_{T}^{2}\right\rangle}} \\
& \times\left[X_{T}^{2}\right\rangle
\end{aligned} .
$$

From the first to the second line of the last two equations we used Wick's theorem and the fact that $X_{t}$ has mean zero. Putting everything together, we arrive at Eqs. (8) and (9).

\section{Appendix B: Abel transform and inversion of $\mathcal{K}_{\frac{1}{2}}$ transform}

For a real function $g(\vartheta)$ non-vanishing on the interval $[0,1]$, we consider the transformation $\mathcal{K}_{\frac{1}{2}}$ defined as

$$
\bar{g}(\kappa) \equiv \mathcal{K}_{\frac{1}{2}}[g](\kappa):=\int_{0}^{1} \frac{g(\vartheta)}{\sqrt{1+\kappa \vartheta}} \mathrm{d} \vartheta
$$

The question is how to reconstruct $g$, knowing $\bar{g}$.

The Abel transform $F$ of a function $f$ is defined as [38, 39]

$$
F(y)=\int_{y}^{\infty} \frac{2 r f(r)}{\sqrt{r^{2}-y^{2}}} \mathrm{~d} r
$$


The inverse formula, allowing to recover $f$ from $F$, is

$$
f(r)=-\frac{1}{\pi} \int_{r}^{\infty} \frac{F^{\prime}(y)}{\sqrt{y^{2}-r^{2}}} \mathrm{~d} y .
$$

To make the link with $\mathcal{K}_{\frac{1}{2}}$, we change variables from $\vartheta$ to $r:=\sqrt{\vartheta}$ in Eq. B1, and introduce $f(r):=g\left(\vartheta=r^{2}\right)$. Then, for $\kappa>0$,

$$
\bar{g}(\kappa)=\int_{0}^{1} \frac{f(r)}{\sqrt{1+\kappa r^{2}}} 2 r \mathrm{~d} r=\frac{2}{\sqrt{\kappa}} \int_{0}^{\infty} \frac{f(r) r}{\sqrt{\frac{1}{\kappa}+r^{2}}} \mathrm{~d} r
$$

In the last equality, we changed the upper integration limit, using $f(r)=0$ for $r>1$. We now continue $\bar{g}(\kappa) \sqrt{\kappa}$ in the complex plane from real positive to real negative $\kappa$, by setting $\kappa=e^{i \varphi} /\left.y^{2}\right|_{\varphi= \pm \pi}$ with $y>0$. This gives

$$
\begin{aligned}
\bar{g}(\kappa) \sqrt{\kappa} & =\int_{y}^{\infty} \frac{2 r f(r)}{\sqrt{r^{2}-y^{2}}} \mathrm{~d} r+\int_{0}^{y} \frac{2 r f(r)}{\sqrt{r^{2}-y^{2}}} \mathrm{~d} r \\
& =F(y)+e^{-i \varphi / 2} G(y) .
\end{aligned}
$$

We have split the integral over $r$ into two parts: the first part is a real function $F(y) \in \mathbb{R}$, which is the Abel transform of $f(r)$. The second term is purely imaginary because of the denominator; which of the two possible branches is taken depends on how we continued $\bar{g}(\kappa) \sqrt{\kappa}$, choosing either of the branches $\varphi= \pm \pi$. This means that we can express the Abel transform $F(y)$ of $f(r)$ from $\bar{g}(\kappa)$ as

$$
F(y)=\mathfrak{R}\left[\left.\bar{g}(\kappa) \sqrt{\kappa}\right|_{\kappa=-1 / y^{2}},\right.
$$

where $\mathfrak{R}$ denotes the real part. We can now use formula $\mathrm{B} 3$ to invert the Abel transform.

Since $f(r)$ vanishes for $r>1$, according to the definition (B2) also $F(y)$ vanishes for $y>1$. One can thus reduce the upper bound in Eq. (B3) to 1. Finally reintroducing the function $g(\vartheta)$ instead of $f(r)$, we get

$$
g(\vartheta)=-\frac{1}{\pi} \int_{\sqrt{\vartheta}}^{1} \frac{F^{\prime}(y)}{\sqrt{y^{2}-\vartheta}} \mathrm{d} y
$$

where $F(y)$ is defined from $\bar{g}(\kappa)$ in Eq. B6. We now want to apply this to compute $g_{1}(\vartheta)$ from Eq. (71). We need to compute the inverse $\mathcal{K}_{1 / 2}$ transform of

$$
\begin{array}{r}
\bar{g}_{1}^{\text {pos }}(\kappa)-\bar{g}_{0,1}(\kappa)=8\left(\frac{1}{\sqrt{\kappa+1}}+1\right) \ln (\sqrt{\kappa+1}+1) \\
-16 \frac{\ln (4)-1}{\sqrt{\kappa+1}+1}-\frac{4(\kappa+\sqrt{\kappa+1}+1) \ln (\kappa+1)}{\kappa} .
\end{array}
$$

From scaling, we expect that close to the boundary

$$
g_{1}(\vartheta) \simeq-2 \ln (\vartheta(1-\vartheta))
$$

To simplify the calculation, we subtract this divergent part. Define

$$
\begin{aligned}
\bar{g}^{\ln }(\kappa):= & \int_{0}^{1} \mathrm{~d} \vartheta \frac{\ln (\vartheta(1-\vartheta))+2}{\sqrt{1+\kappa \vartheta}} \\
= & \frac{4[\ln (2)-1]}{1+\sqrt{\kappa+1}+\frac{2 \sqrt{\kappa+1} \ln (\kappa+1)}{\kappa}} \\
& +\frac{4(1-\sqrt{\kappa+1}) \ln (\sqrt{\kappa+1}+1)}{\kappa} .
\end{aligned}
$$

Setting $\bar{g}(\kappa):=\bar{g}_{1}^{\text {pos }}(\kappa)-\bar{g}_{0,1}(\kappa)+2 \bar{g}^{\ln }(\kappa)$ in Eq. (B6) yields

$$
\begin{aligned}
F(y)= & -\frac{8 y^{2} \ln (y)}{\sqrt{1-y^{2}}}-24 \sqrt{1-y^{2}} \ln (2) \\
& -\frac{8\left(y^{2}-1\right) \arcsin (y)}{y} .
\end{aligned}
$$

Computing the integral (B7) finally gives

$$
\begin{aligned}
g(\vartheta)= & \mathcal{K}_{\frac{1}{2}}^{-1}\left[\bar{g}_{1}(\kappa)-\bar{g}_{0,1}(\kappa)+2 \bar{g}^{\ln }(\kappa)\right] \\
=4 & {\left[3-\frac{1}{\sqrt{1-\vartheta}+1}-\frac{1}{\sqrt{\vartheta}+1}\right.} \\
& \left.+\ln \left(\frac{(\sqrt{\vartheta}+1)(\sqrt{1-\vartheta}+1)}{16}\right)\right] .
\end{aligned}
$$

Adding the logarithmic terms, we recover the result $(73)$ given in the main text.

\section{Appendix C: Inverse Laplace transforms necessary for the maximum of the bridge, and other useful relations}

In this appendix we give a table of useful relations for the inverse Laplace transforms encountered in this article.

All appearing hypergeometric functions can be eliminated by using two special functions, introduced in Refs. [32-34], and named $\mathcal{I}(x)$ and $\mathcal{J}(x)$,

$$
\begin{aligned}
\mathcal{I}(x) & =\frac{1}{6} x^{4}{ }_{2} F_{2}\left(1,1 ; \frac{5}{2}, 3 ; \frac{x^{2}}{2}\right)+\pi\left(1-x^{2}\right) \operatorname{erfi}\left(\frac{x}{\sqrt{2}}\right) \\
& +\sqrt{2 \pi} e^{\frac{x^{2}}{2}} x+2-3 x^{2}, \\
\mathcal{J}(x) & =\frac{1}{2} \pi \operatorname{erfi}(\sqrt{x})-x_{2} F_{2}\left(1,1 ; \frac{3}{2}, 2 ; x\right)
\end{aligned}
$$

These functions are related to each other by the relations

$$
\begin{aligned}
& \mathcal{I}(x)=2+2\left(1-x^{2}\right) \mathcal{J}\left(\frac{x^{2}}{2}\right)+\sqrt{2 \pi} e^{\frac{x^{2}}{2}} x \operatorname{erfc}\left(\frac{x}{\sqrt{2}}\right), \\
& \mathcal{I}(x)=-2 e^{\frac{x^{2}}{2}} \partial_{x}^{2}\left[e^{-\frac{x^{2}}{2}} \mathcal{J}\left(\frac{x^{2}}{2}\right)\right] .
\end{aligned}
$$

To arrive at these identities, and to express everything in terms of one of these two functions, two non-trivial relations 
between hypergeometric functions were used (they can be checked by Taylor-expansion to high order)

$$
\begin{gathered}
-3_{2} F_{2}\left(1,1 ; \frac{3}{2}, 2 ; \frac{x^{2}}{2}\right)+{ }_{2} F_{2}\left(1,1 ; 2, \frac{5}{2} ; \frac{x^{2}}{2}\right) \\
+\frac{6}{x^{2}}\left[\sqrt{\frac{\pi}{2}} \frac{e^{\frac{x^{2}}{2}}}{x} \operatorname{erf}\left(\frac{x}{\sqrt{2}}\right)-1\right]=0 \\
-x^{3}\left[3{ }_{2} F_{2}\left(1,1 ; \frac{3}{2}, 2 ;-\frac{x^{2}}{2}\right)+{ }_{2} F_{2}\left(1,1 ; 2, \frac{5}{2} ; \frac{x^{2}}{2}\right)\right] \\
+\operatorname{erf}\left(\frac{x}{\sqrt{2}}\right)\left[3 \pi x \operatorname{erfi}\left(\frac{x}{\sqrt{2}}\right)-3 \sqrt{2 \pi} e^{\frac{x^{2}}{2}}\right]+6 x=0 .
\end{gathered}
$$

We now express the needed inverse Laplace transforms either in terms of $\mathcal{I}$ or $\mathcal{J}$, depending on which form is more compact. (Note that each function appears naturally in a given context [34]).

Transforms involving only $e^{-\sqrt{s}}$, and powers of $\sqrt{s}$ are elementary,

$$
\begin{aligned}
\mathcal{L}_{s \rightarrow t}^{-1}\left[e^{-\sqrt{s}}\right] & =\frac{e^{-\frac{1}{4 t}}}{2 \sqrt{\pi} t^{3 / 2}} \\
\mathcal{L}_{s \rightarrow t}^{-1}\left[e^{-\sqrt{s}} \sqrt{s}\right] & =-\frac{e^{-\frac{1}{4 t}}(2 t-1)}{4 \sqrt{\pi} t^{5 / 2}} \\
\mathcal{L}_{s \rightarrow t}^{-1}\left[\frac{e^{-\sqrt{s}}}{\sqrt{s}}\right] & =\frac{e^{-\frac{1}{4 t}}}{\sqrt{\pi t}} .
\end{aligned}
$$

Transforms with an additional factor of $\ln (s)$ are

$$
\begin{aligned}
\mathcal{L}_{s \rightarrow t}^{-1}\left[e^{-\sqrt{s}} \sqrt{s} \ln (s)\right] & =-\frac{e^{-\frac{1}{4 t}}}{4 \sqrt{\pi} t^{5 / 2}}\left\{-2 t \mathcal{I}\left(\frac{1}{\sqrt{2 t}}\right)+(2 t-1)\left[\ln (4 t)+\gamma_{\mathrm{E}}\right]\right\} \\
\mathcal{L}_{s \rightarrow t}^{-1}\left[\frac{e^{-\sqrt{s}} \ln (s)}{\sqrt{s}}\right] & =\frac{e^{-\frac{1}{4 t}}}{\sqrt{\pi t}}\left[2 \mathcal{J}\left(\frac{1}{4 t}\right)-\ln (4 t)-\gamma_{\mathrm{E}}\right] \\
\mathcal{L}_{s \rightarrow t}^{-1}\left[e^{-\sqrt{s}} \ln (s)\right] & =\frac{e^{-\frac{1}{4 t}}}{4 \sqrt{\pi} t^{5 / 2}}\left[2 \mathcal{J}\left(\frac{1}{4 t}\right)-\ln (4 t)-\gamma_{\mathrm{E}}\right]-\frac{\operatorname{erfc}\left(\frac{1}{2 \sqrt{t}}\right)}{t} .
\end{aligned}
$$

Transforms involving the exponential integral function are

$$
\begin{aligned}
\mathcal{L}_{s \rightarrow t}^{-1}[\operatorname{Ei}(-\sqrt{s})] & =-\frac{\operatorname{erfc}\left(\frac{1}{2 \sqrt{t}}\right)}{2 t} \\
\mathcal{L}_{s \rightarrow t}^{-1}\left[e^{\sqrt{s}} \operatorname{Ei}(-2 \sqrt{s})\right] & =\frac{e^{-\frac{1}{4 t}}}{4 \sqrt{\pi} t^{3 / 2}}\left[2 \mathcal{J}\left(\frac{1}{4 t}\right)+\ln (t)-\gamma_{\mathrm{E}}\right]-\frac{\operatorname{erfc}\left(\frac{1}{2 \sqrt{t}}\right)}{2 t} \\
\mathcal{L}_{s \rightarrow t}^{-1}\left[\sqrt{s} e^{\sqrt{s}} \operatorname{Ei}(-2 \sqrt{s})\right] & =\frac{e^{-\frac{1}{4 t}}}{8 \sqrt{\pi} t^{5 / 2}}\left\{2 t \mathcal{I}\left(\frac{1}{\sqrt{2 t}}\right)+(2 t-1)\left[\ln (t)-\gamma_{\mathrm{E}}\right]-8 t\right\} \\
\mathcal{L}_{s \rightarrow t}^{-1}\left[\frac{e^{\sqrt{s}} \operatorname{Ei}(-2 \sqrt{s})}{\sqrt{s}}\right] & =\frac{e^{-\frac{1}{4 t}}}{2 \sqrt{\pi t}}\left[\gamma_{\mathrm{E}}-2 \mathcal{J}\left(\frac{1}{4 t}\right)-\ln (t)\right] .
\end{aligned}
$$

[1] A. Einstein, Über die von der molekularkinetischen Theorie der Wärme geforderte Bewegung von in ruhenden Flüssigkeiten suspendierten Teilchen, Annalen der Physik 322 (1905) 549_ 560.

[2] W. Feller, Introduction to Probability Theory and Its Applications, John Wiley \& Sons, 1950.

[3] J.-P. Bouchaud and A. Georges, Anomalous diffusion in disordered media: statistical mechanisms, models and physical applications, Phys. Rep. 195 (1990) 127-293.

[4] J.-C. Walter, A. Ferrantini, E. Carlon and C. Vanderzande, Fractional Brownian motion and the critical dynamics of zipping polymers, Phys. Rev. E 85 (2012) 031120.

[5] A. Amitai, Y. Kantor and M. Kardar, First-passage distribu- tions in a collective model of anomalous diffusion with tunable exponent, Phys. Rev. E 81 (2010) 011107.

[6] A. Zoia, A. Rosso and S.N. Majumdar, Asymptotic behavior of self-affine processes in semi-infinite domains, Phys. Rev. Lett. 102 (2009) 120602.

[7] J. L. A. Dubbeldam, V. G. Rostiashvili, A. Milchev and T. A. Vilgis, Fractional Brownian motion approach to polymer translocation: The governing equation of motion, Phys. Rev. E 83 (2011) 011802.

[8] V. Palyulin, T. Ala-Nissila and R. Metzler, Polymer translocation: the first two decades and the recent diversification, Soft Matter 10 (2014) 9016-9037.

[9] P. L. Krapivsky, K. Mallick and T. Sadhu, Large deviations in 
single-file diffusion, Phys. Rev. Lett. 113 (2014) 078101.

[10] P. L. Krapivsky, K. Mallick and T. Sadhu, Dynamical properties of single-file diffusion, arXiv:1505.01287 (2015).

[11] P. L. Krapivsky, K. Mallick and T. Sadhu, Tagged particle in single-file diffusion, J. Stat. Phys. 160 (2015) 885-925.

[12] V. Kukla, J. Kornatowski, D. Demuth, I. Girnus, H. Pfeifer, L.V.C. Rees, S. Schunk, K.K. Unger and J. Karger, NMR studies of single-file diffusion in unidimensional channel zeolites, Science (1996) 702.

[13] Q.-H. Wei, C. Bechinger and P. Leiderer, Single-file diffusion of colloids in one-dimensional channels, Science 287 (2000) 625-627.

[14] S. Gupta, A. Rosso and C. Texier, Dynamics of a tagged monomer: Effects of elastic pinning and harmonic absorption, Phys. Rev. Lett. 111 (2013) 210601.

[15] D. Panja, Probabilistic phase space trajectory description for anomalous polymer dynamics, Journal of Physics: Condensed Matter 23 (2011) 105103.

[16] N.J. Cutland, P.E. Kopp and W. Willinger, Stock price returns and the Joseph effect: A fractional version of the Black-Scholes model, in E. Bolthausen, M. Dozzi and F. Russo, editors, Seminar on Stochastic Analysis, Random Fields and Applications, Volume 36 of Progress in Probability, pages 327-351, Birkhäuser Basel, 1995.

[17] L.C.G. Rogers, Arbitrage with fractional Brownian motion, Mathematical Finance 7 (1997) 95-105.

[18] S. Rostek and R. Schöbel, A note on the use of fractional Brownian motion for financial modeling, Economic Modelling 30 (2013) $30-35$.

[19] B.B. Mandelbrot and J.R. Wallis, Noah, Joseph, and operational hydrology, Water Resources Research 4 (1968) 909-918.

[20] F.J. Molz, H.H. Liu and J. Szulga, Fractional Brownian motion and fractional Gaussian noise in subsurface hydrology: A review, presentation of fundamental properties, and extensions, Water Resources Research 33 (1997) 2273-2286.

[21] Y.G. Sinai, Distribution of the maximum of a fractional Brownian motion, Russian Math. Surveys 52 (1997) 359-378.

[22] S.N. Majumdar, Persistence in nonequilibrium systems, Curr. Sci. 77 (1999) 370.

[23] G. M. Molchan, Maximum of a fractional Brownian motion: Probabilities of small values, Communications in Mathematical Physics 205 (1999) 97-111.

[24] I. Nourdin, Selected Aspects of Fractional Brownian Motion, Bocconi \& Springer Series, 2012.

[25] F. Aurzada, On the one-sided exit problem for fractional Brownian motion, Electron. Commun. Probab. 16 (2011) no. 36, 392-404.

[26] K. Oerding, S. J. Cornell and A. J. Bray, Non-markovian persistence and nonequilibrium critical dynamics, Phys. Rev. E 56 (1997) R25-R28.

[27] A. B. Dieker, Simulation of fractional Brownian motion, PhD thesis, University of Twente, 2004.

[28] L.B.G. Andersen and V.V. Piterbarg, Interest Rate Modeling. Volume 1: Foundations and Vanilla Models, Atlantic Financial Press, 2010.

[29] A.W. Van der Vaart, Asymptotic Statistics, Cambridge University Press, 1998.

[30] P. Lévy, Sur certains processus stochastiques homogènes, Compositio Mathematica 7 (1940) 283-339.
[31] Y. Nikitin and E. Orsingher, The intermediate arc-sine law, Stat. \& Proba. Letters 49 (2000) 119 - 125.

[32] K.J. Wiese, S.N. Majumdar and A. Rosso, Perturbation theory for fractional Brownian motion in presence of absorbing boundaries, Phys. Rev. E 83 (2011) 061141, arXiv:1011.4807.

[33] M. Delorme and K.J. Wiese, The maximum of a fractional Brownian motion: Analytic results from perturbation theory, Phys. Rev. Lett. 115 (2015) 210601, arXiv:1507.06238.

[34] M. Delorme and K.J. Wiese, Perturbative expansion for the maximum of fractional Brownian motion, arXiv:1603.00651 (2016).

[35] M. Delorme and K.J. Wiese, unpublished.

[36] T. Sottinen D. Gasbarra and E. Valkeila, Gaussian bridges, Abel Symposium (2005).

[37] J. Bertrand, Solution d'un problème, Comptes Rendus de l'Académie des Sciences, Paris 105 (1887) 369.

[38] N. H. Abel, Auflösung einer mechanischen Aufgabe, Journal für Die Reine und Angewandte Mathematik 1 (1826) 153-157.

[39] R. Bracewell, The Fourier Transform and its Applications, McGraw-Hill, New York, 1965.

\section{CONTENTS}

I. Introduction

II. Preliminaries: Gaussian Bridges

III. Pertubative approach to $\mathrm{fBm}$

IV. Positive time of a fBm Bridge
A. Positive time of a discrete random walk
B. Propagators in continuous time
C. Scale invariance and a useful transformation
D. FBm bridge with $H=\frac{1}{2}+\varepsilon$
E. Numerical results

V. Extremum of $\mathrm{fBm}$ Bridges

9
10
11
12

VI. Conclusions

13

VII. Acknowledgments

13

A. Details on correlation functions for the bridge 13

B. Abel transform and inversion of $\mathcal{K}_{\frac{1}{2}}$ transform

C. Inverse Laplace transforms necessary for the maximum of the bridge, and other useful relations

References

15 